\title{
UNITARIZATION OF LOOP GROUP REPRESENTATIONS OF FUNDAMENTAL GROUPS
}

\author{
JOSEF DORFMEISTER AND HONGYOU WU
}

\begin{abstract}
In this paper, we give a characterization of the simultaneous unitarizability of any finite set of $\operatorname{SL}(2, \mathbb{C})$-valued functions on $\mathbb{S}^{1}$ and determine all possible ways of the unitarization. Such matrix functions can be regarded as images of the generators for the fundamental group of a surface in an $\mathbb{S}^{1}$-family, and the results of this paper have applications in the construction of constant mean curvature surfaces in space.
\end{abstract}

In recent years, a Weierstrass type representation [4] has been used to construct surfaces of non-zero constant mean curvature in space (CMC surfaces). This construction starts with an $\operatorname{sl}(2, \mathbb{C})$-valued holomorphic or meromorphic 1-form on some (compact or non-compact) Riemann surface $\mathcal{S}$ other than $\mathbb{S}^{2}$. Pulling back to the simply connected cover $\widetilde{\mathcal{S}}$ of $\mathcal{S}$, one obtains a 1 -form $\eta$ on $\widetilde{\mathcal{S}}$ which is invariant under the fundamental group $\pi_{1}(\mathcal{S})$ of $\mathcal{S}$. In the next step of the construction, one solves the ordinary differential equation $\mathrm{d} H=H \eta$ on $\widetilde{\mathcal{S}}$ with the initial condition $I$ at some base point $z_{0}$. Then, every $\gamma \in \pi_{1}(\mathcal{S})$ induces a monodromy matrix $\rho_{\gamma}$ via $H$. As a special feature of this construction, everything depends also on a parameter $\lambda \in \mathbb{S}^{1}$. In particular, for $\gamma \in \pi_{1}(\mathcal{S})$, the matrix $\rho_{\gamma}=\rho_{\gamma}(\lambda)$ depends holomorphically on $\lambda$ on $\mathbb{C}^{*}=\mathbb{C} \backslash\{0\}$. In this way, one obtains a map $\rho$ from $\pi_{1}(\mathcal{S})$ into the loops in $\operatorname{SL}(2, \mathbb{C})$ satisfying $\rho_{\gamma \delta}=\rho_{\gamma} \rho_{\delta}$, i.e., $\rho$ is a representation of $\pi_{1}(\mathcal{S})$ in the $\mathrm{SL}(2, \mathbb{C})$-loop group. When the CMC surface to be constructed has umbilic points, in order to obtain eventually an immersion from $\mathcal{S}$ into $\mathbb{R}^{3}$, not just from $\widetilde{\mathcal{S}}$ into $\mathbb{R}^{3}$, it is necessary that $\rho_{\gamma}(\lambda) \in \mathrm{SU}(2)$ for all $\gamma \in \pi_{1}(\mathcal{S})$ and $\lambda \in \mathbb{S}^{1}$. At this point, no criterion on $\eta$ is known which would characterize the 1 -forms on $\widetilde{\mathcal{S}}$ corresponding to unitary representations $\rho$. Therefore, one tries to find conditions under which one can conjugate $\rho$ such that the new representation $\hat{\rho}$ is unitary. The new representation $\hat{\rho}$ can always be derived from some 1-form $\hat{\eta}$, which can be obtained from $\eta$ by a gauge transformation. This actually corresponds to

Received July 14, 2005.

2000 Mathematics Subject Classification: Primary 22E67; Secondary 53A10, 53C42. 
using an initial condition at $z_{0}$ different from $I$ when solving the differential equation $\mathrm{d} H=H \eta$.

In this paper, we present an algorithm testing and characterizing the unitarizability of a monodromy representation $\rho$. It suffices to check whether a set of generators $L_{1}, L_{2}, \ldots, L_{m}$ of the image of $\pi_{1}(\mathcal{S})$ under $\rho$ can be simultaneously unitarized. It turns out that this is possible only if each $L_{i}$ has an eigenvalue function $v_{i}(\lambda)$ which is holomorphic on a neighborhood of $\mathbb{S}^{1}$. We will also assume that no $L_{i}$ has the $\lambda$-independent eigenvalues \pm 1 , which is satisfied in the geometric context mentioned above. Then, the unitarizability of one matrix function $L$ can be characterized by the obvious conditions on the trace of $L$, see Proposition 2.2. Also, a specific matrix function $T$ can be found conjugating $L$ into some canonical unitary matrix function. Given two matrix functions $L_{1}$ and $L_{2}$ satisfying these conditions and thus being unitarizable separately by some $T_{1}$ and $T_{2}$, respectively, we first consider the case where $L_{1}$ and $L_{2}$ commute. This can be characterized by $T_{1} T_{2}^{-1}$ being diagonal or off-diagonal, see Proposition 3.1. Since commuting with each other is an equivalence relation, we can assume that no pair of the generators $L_{1}, L_{2}, \ldots, L_{m}$ commute. In this case, we characterize the simultaneous unitarizability of $L_{1}$ and $L_{2}$ by simple conditions on the entry functions of $T_{1} T_{2}^{-1}$, see Theorem 3.5. Similarly, the simultaneous unitarizability of $L_{1}, L_{2}$ and $L_{3}$ is characterized by simple conditions on the entry functions of $T_{1} T_{2}^{-1}$ and $T_{2} T_{3}^{-1}$, with each $T_{i}$ having the same meaning as above, see Theorem 4.1. Finally, for the case of four or more generators, it turns out that it suffices to verify that every three consecutive generators are simultaneously unitarizable, see Theorem 4.14 .

In the geometric applications to CMC surfaces mentioned above, one actually uses the twisted loop group consisting of the loops in $\mathrm{SL}(2, \mathbb{C})$ which reflect some invariance under the Cartan involution of $\mathrm{SL}(2, \mathbb{C})$ corresponding to the symmetric space $\mathbb{S}^{2}=\mathrm{SU}(2) / \mathrm{U}(1)$. Therefore, it is natural that in view of these applications, we use twisted loop groups throughout this paper. However, there is also some technical advantage: while the twisted and untwisted loop groups are isomorphic in the case of $\operatorname{SL}(2, \mathbb{C})$, this isomorphism does not preserve the so-called "positive loops", see (1.10). Thus, writing this paper in the untwisted setting would require replacing frequently used standard loop group splittings (Birkhoff and Iwasawa splittings) by closely related ones, for which at least some of the properties would need to be proven. Finally, from the point of view of Kac-Moody groups, twisted and untwisted groups are equally natural, and the untwisted version should not 
be preferred.

Another issue is how to deal with cases where some eigenvalue function vanishes at some points of $\mathbb{S}^{1}$. Since these eigenvalue functions do not vanish identically on $\mathbb{S}^{1}$ and are holomorphic in some neighborhood of $\mathbb{S}^{1}$, it is natural to consider $r$-circles which are sufficiently close to $\mathbb{S}^{1}$. Fortunately, for these $r$-loops, there is already a well-developed theory for us to use.

It is worth pointing out that up to conjugations by unitary matrix functions, there is at most one way to simultaneously unitarize two or more matrix functions, unless they commute for all values of $\lambda \in \mathbb{S}^{1}$, see Theorems 3.5, 3.24, 4.1 and 4.14. This result will be essential in proving the so-called "closing conditions" when $r$-dressing with $r<1$ is needed. In the geometric applications discussed above, these closing conditions ensure that the CMC immersion from $\widetilde{\mathcal{S}}$ into $\mathbb{R}^{3}$ constructed from $\eta$ actually descends down to $\mathcal{S}$ and yields a CMC immersion from $\mathcal{S}$ into $\mathbb{R}^{3}$.

We mention that it seems more natural to consider the unitarization problem in the algebra of matrix functions meromorphic in a neighborhood of $\mathbb{S}^{1}$. However, in general, the problem does not have a solution in that algebra, see the proof of Theorem 2.6 together with Remark 2.14. Moreover, the topology on the Wiener algebra needed for the geometric applications that we are primarily interested in adds severe difficulties to the problem.

This paper is organized as follows. In Section 1, we introduce the basic definitions concerning loop groups and recall some fundamental results. In Section 2, we characterize the unitarizability of one matrix function $L$ and determine all the matrix functions unitarizing $L$. Section 3 is devoted to the characterization of the simultaneous unitarizability of two matrix functions, while Section 4 considers three or more matrix functions. Finally, in Section 5, we discuss the relation between the simultaneous unitarizability of matrix functions by dressing and their pointwise simultaneous unitarizability, and deal with the closing conditions.

\section{§1. Notation and basic results on loop groups}

In this paper, for any $r>0$ and each $c \in \mathbb{C}$, we will use $\mathbb{S}_{r}^{1}(c), \mathcal{D}_{r}(c)$ and $\mathcal{D}_{r}^{*}(c)$ to denote the circle $\{z \in \mathbb{C} ;|z-c|=r\}$, the disk $\{z \in \mathbb{C} ;|z-c|<r\}$ and the punctured disk $\{z \in \mathbb{C} ; 0<|z-c|<r\}$, respectively. When any of $c=0$ and $r=1$ happens, we will omit the corresponding part from the notation: $\mathbb{S}^{1}=\mathbb{S}_{1}^{1}=\mathbb{S}_{1}^{1}(0), \mathcal{D}=\mathcal{D}_{1}=\mathcal{D}_{1}(0)$, etc.

First, we state a result about holomorphic functions. This result will be needed in Sections 2 and 3. 
LEMMA 1.1. If a function $f$ is even, holomorphic on a neighborhood of $\mathbb{S}_{r}^{1}$ and real-valued on $\mathbb{S}_{r}^{1}$, and any zero of $f$ on $\mathbb{S}_{r}^{1}$ has an even order, then there is a function $g$ such that $g^{2}=f$ and $g$ is also holomorphic on a neighborhood of $\mathbb{S}_{r}^{1}$, and each such $g$ is either even in $\lambda$ or odd in $\lambda$.

Proof. Note that either $f \geq 0$ on $\mathbb{S}_{r}^{1}$ or $f \leq 0$ on $\mathbb{S}_{r}^{1}$. Without loss of generality, we assume further that $f \geq 0$ on $\mathbb{S}_{r}^{1}$. Locally, $f$ has two holomorphic square roots which are real at points of $\mathbb{S}_{r}^{1}$. If they change sign at a zero $\lambda_{0} \in \mathbb{S}_{r}^{1}$ of $f$, then the corresponding local holomorphic square roots near $-\lambda_{0}$ also change sign at $-\lambda_{0}$, since $f$ is even in $\lambda$. Thus, local holomorphic square roots can be extended to become global holomorphic square roots on a neighborhood of $\mathbb{S}_{r}^{1}$ : consider a $\lambda_{*} \in \mathbb{S}_{r}^{1}$ which is not a zero of $f$, and along $\mathbb{S}_{r}^{1}$ extend holomorphically the positive local holomorphic square root near $\lambda_{*}$, then the extension is still positive at $\lambda_{*}$ (and hence agrees with the original square root at $\lambda_{*}$ ) when $\mathbb{S}_{r}^{1}$ is exhausted. Note that two local holomorphic square roots of $f$ only differ by a sign. Since again $f$ is even in $\lambda, g$ must be either even in $\lambda$ or is odd in $\lambda$.

We denote by $\mathcal{W}_{r}$ the Wiener algebra [8] on $\mathbb{S}_{r}^{1}$, i.e., the Banach algebra of continuous functions $f$ on $\mathbb{S}_{r}^{1}$ whose Fourier expansion $f(\lambda)=\sum_{i \in \mathbb{Z}} f_{i} \lambda^{i}$ exists and satisfies

$$
\|f\|_{r}:=\sum_{i \in \mathbb{Z}}\left|f_{i}\right| r^{i}<+\infty .
$$

Moreover, $\mathcal{W}_{r}^{*}$ stands for the subalgebra of $\mathcal{W}_{r}$ consisting of elements which never vanish on $\mathbb{S}_{r}^{1}, \mathcal{W}_{r}^{-}$the subalgebra consisting of elements whose Fourier expansion has only negative powers of $\lambda$, and $\mathcal{W}_{r}^{+}$the subalgebra consisting of elements whose Fourier expansion does not involve any negative power of $\lambda$. We will need the following results later.

LEMmA 1.3. Let $n \in \mathbb{N}$. If $h \in \mathcal{W}_{r}^{*}$ is always positive on $\mathbb{S}_{r}^{1}$, then there is a $k_{+} \in \mathcal{W}_{r}^{+}$never vanishing on $\mathcal{D}_{r}$ and satisfying

$$
h(\lambda)=k_{+}(\lambda)^{n} \overline{k_{+}\left(r^{2} / \bar{\lambda}\right)^{n}} \quad \text { on } \mathbb{S}_{r}^{1}
$$

Moreover, if $h$ is even in $\lambda$, then so is $k_{+}$; if $h(-\lambda)=1 / h(\lambda)$ for all $\lambda \in \mathbb{S}_{r}^{1}$, then $k_{+}=\mathrm{e}^{\alpha_{+}}$for some $\alpha_{+} \in \mathcal{W}_{r}^{+}$which is odd in $\lambda$. 
Proof. By the Birkhoff decomposition on $\mathbb{S}_{r}^{1}[6]$,

$$
h(\lambda)=c \lambda^{m} h_{-}(\lambda) h_{+}(\lambda) \quad \text { on } \mathbb{S}_{r}^{1},
$$

where $c$ is a non-zero constant, $m \in \mathbb{Z}, h_{-} \in \mathcal{W}_{r}^{-}$never vanishes on $\overline{\mathcal{E}_{r}}:=$ $\{|\lambda| \geq r\} \cup\{\infty\}$ and satisfies $h_{-}(\infty)=1$, while $h_{+} \in \mathcal{W}_{r}^{+}$never vanishes on the closure $\overline{\mathcal{D}_{r}}$ of $\mathcal{D}_{r}$ and satisfies $h_{+}(0)=1$. By the uniqueness of the Birkhoff decomposition, the reality of $h$ on $\mathbb{S}_{r}^{1}$ is equivalent to that

$$
c \in \mathbb{R}, \quad m=0, \quad h_{-}(\lambda)=\overline{h_{+}(\lambda)}=\overline{h_{+}\left(r^{2} / \bar{\lambda}\right)} \quad \text { on } \mathbb{S}_{r}^{1} .
$$

So, $c>0$ by the positivity of $h$ on $\mathbb{S}_{r}^{1}$. Since $h_{+} \in \mathcal{W}_{r}^{+}$never vanishes on $\overline{\mathcal{D}_{r}}$, it has an $n$-th root in $\mathcal{W}_{r}^{+}$, say $l_{+}$. Therefore, we can set $k_{+}=c^{\prime} l_{+}$, where $c^{\prime}>0$ is a $2 n$-th root of $c$.

If $h$ is even in $\lambda$, then (1.4) and the uniqueness of the Birkhoff decomposition yield the evenness of $k_{+}$. If $h(-\lambda)=1 / h(\lambda)$ for all $\lambda \in \mathbb{S}_{r}^{1}$, then $k_{+}(\lambda) k_{+}(-\lambda)=1$, and hence $k_{+}=\mathrm{e}^{\alpha_{+}}$for some $\alpha_{+} \in \mathcal{W}_{r}^{+}$which is odd in $\lambda$.

We will frequently use the following relation between the Wiener algebra and the set of analytic functions: let $0 \leq r<R \leq+\infty$, then a function on $\{\lambda \in \mathbb{C} ; r<|\lambda|<R\}$ is in $\mathcal{W}_{s}$ for all $s \in(r, R)$ if and only if it is analytic on the annulus.

Set $\boldsymbol{G}=\mathrm{SU}(2)$. Then, the complexification of $\boldsymbol{G}$ is $\boldsymbol{G}^{\mathbb{C}}=\mathrm{SL}(2, \mathbb{C})$. Let $r \in(0,+\infty)$. We introduce the following twisted $r$-loop groups (see [4], [2] and [3] for details), in which $\lambda \in \mathbb{S}_{r}^{1}$ serves as the loop parameter:

$$
\begin{aligned}
& \Lambda_{r} \boldsymbol{G}_{\mathrm{t}}^{\mathbb{C}}=\left\{G: \mathbb{S}_{r}^{1} \rightarrow \boldsymbol{G}^{\mathbb{C}} ; \begin{array}{r}
g_{i j} \in \mathcal{W}_{r} \text { for each } i, j \\
g_{11}, g_{22} \text { are even in } \lambda \\
g_{12}, g_{21} \text { are odd in } \lambda
\end{array}\right\}, \\
& \Lambda_{r} \boldsymbol{G}_{\mathrm{t}}=\left\{G \in \Lambda_{r} \boldsymbol{G}_{\mathrm{t}}^{\mathbb{C}} ; \begin{array}{l}
G \text { has a continuous extension } \\
\text { up to } \mathbb{S}^{1} \text { and is unitary on } \mathbb{S}^{1}
\end{array}\right\}, \\
& \Lambda_{r, *}^{-} \boldsymbol{G}_{\mathrm{t}}^{\mathbb{C}}=\left\{G \in \Lambda_{r} \boldsymbol{G}_{\mathrm{t}}^{\mathbb{C}} ; \begin{array}{l}
G \text { extends holomorphically } \\
\text { to } \mathcal{E}_{r}, \text { and } G(\infty)=I
\end{array}\right\}, \\
& \Lambda_{r}^{+} \boldsymbol{G}_{\mathrm{t}}^{\mathbb{C}}=\left\{G \in \Lambda_{r} \boldsymbol{G}_{\mathrm{t}}^{\mathbb{C}} ; G \text { extends holomorphically to } \mathcal{D}_{r}\right\}, \\
& \Lambda_{r, \boldsymbol{B}}^{+} \boldsymbol{G}_{\mathrm{t}}^{\mathbb{C}}=\left\{G \in \Lambda_{r}^{+} \boldsymbol{G}_{\mathrm{t}}^{\mathbb{C}} ; G(0) \in \boldsymbol{B}\right\},
\end{aligned}
$$

where the $g_{j k}$ 's are the entries of $G, \mathcal{E}_{r}=\{z \in \mathbb{C} ;|z|>r\} \cup\{\infty\}$ and $\boldsymbol{B}$ is the solvable subgroup

$$
\left\{\left(\begin{array}{cc}
\mathrm{e}^{s} & 0 \\
0 & \mathrm{e}^{-s}
\end{array}\right) ; s \in \mathbb{R}\right\}
$$


of $\boldsymbol{G}^{\mathbb{C}}$. Then $\Lambda_{r} \boldsymbol{G}_{\mathrm{t}}^{\mathbb{C}}$ is a complex Banach Lie group. Note that the map

$$
G(\lambda) \longmapsto \overline{G(1 / \bar{\lambda})}
$$

is a Lie group isomorphism from $\Lambda_{r} \boldsymbol{G}_{\mathrm{t}}$ onto $\Lambda_{1 / r} \boldsymbol{G}$, and each element of them is holomorphic in $\lambda$ on the open annulus between $\mathbb{S}_{r}^{1}$ and $\mathbb{S}_{1 / r}^{1}$. The Lie algebra of $\Lambda_{r} \boldsymbol{G}_{\mathrm{t}}^{\mathbb{C}}$ is denoted by $\Lambda_{r} \mathcal{G}^{\mathbb{C}}$, and one has similar notation for the Lie algebras of the other Lie groups defined above. When $r=1$, we will always omit the corresponding subindex. The following results are from Theorem 2.3 of [4].

THEOREM 1.14. The multiplication $\Lambda_{r} \boldsymbol{G}_{\mathrm{t}} \times \Lambda_{r, \boldsymbol{B}}^{+} \boldsymbol{G}_{\mathrm{t}}^{\mathbb{C}} \rightarrow \Lambda_{r} \boldsymbol{G}_{\mathrm{t}}^{\mathbb{C}}$ is an onto diffeomorphism. The multiplication $\Lambda_{r, *}^{-} \boldsymbol{G}_{\mathrm{t}}^{\mathbb{C}} \times \Lambda_{r}^{+} \boldsymbol{G}_{\mathrm{t}}^{\mathbb{C}} \rightarrow \Lambda_{r} \boldsymbol{G}_{\mathrm{t}}^{\mathbb{C}}$ is a diffeomorphism onto the open and dense subset $\Lambda_{r, *}^{-} \boldsymbol{G}_{\mathrm{t}}^{\mathbb{C}} \cdot \Lambda_{r}^{+} \boldsymbol{G}_{\mathrm{t}}^{\mathbb{C}}$.

The splitting $\Lambda_{r} \boldsymbol{G}_{\mathrm{t}}^{\mathbb{C}}=\Lambda_{r} \boldsymbol{G}_{\mathrm{t}} \times \Lambda_{r, \boldsymbol{B}}^{+} \boldsymbol{G}_{\mathrm{t}}^{\mathbb{C}}$ will be called the $r$-Iwasawa splitting, while the splitting $\Lambda_{r, *}^{-} \boldsymbol{G}_{\mathrm{t}}^{\mathbb{C}} \cdot \Lambda_{r}^{+} \boldsymbol{G}_{\mathrm{t}}^{\mathbb{C}}=\Lambda_{r, *}^{-} \boldsymbol{G}_{\mathrm{t}}^{\mathbb{C}} \times \Lambda_{r}^{+} \boldsymbol{G}_{\mathrm{t}}^{\mathbb{C}} \subseteq \Lambda_{r} \boldsymbol{G}_{\mathrm{t}}^{\mathbb{C}}$ is usually called the $r$-Birkhoff splitting. Both of these splittings are analytic. The elements of $\Lambda_{r} \boldsymbol{G}_{\mathrm{t}}$ will sometimes be called unitary $r$-loops or said to be $r$-unitary. When $r=1$, we will also always omit the corresponding prefix.

Remark 1.15. If $r \in(0,1), G \in \Lambda_{r} G_{\mathrm{t}}^{\mathbb{C}}$ is holomorphic on $\mathcal{D}_{r} \backslash \overline{\mathcal{D}_{\rho}}$ for some $\rho \in(0, r)$ and $G=G_{\mathrm{u}} G_{+}$is its $r$-Iwasawa splitting, then $G_{\mathrm{u}}$ is holomorphic on $\mathcal{D}_{1 / \rho} \backslash \overline{\mathcal{D}_{\rho}}$.

The untwisted loop group $\Lambda_{r} G^{\mathbb{C}}$ can be defined by removing the evenodd conditions in (1.7), and its corresponding subgroups can be introduced analogously. We omit the details.

\section{$\S 2$. Unitarization of one loop matrix}

In this section, we give conditions for the unitarizability of one loop matrix and determine all the possible ways to do the unitarization. As pointed out in the introduction, the results on all the possible ways for unitarizing one loop matrix will be used in Sections 3 and 4 in the unitarization of two or more loop matrices. We also give examples showing that in general, it is necessary to use $r$-dressing with $r<1$ for unitarization.

Let $r \in(0,1]$. We will use the notation $\Lambda \boldsymbol{G}_{\mathrm{t}}^{\mathbb{C}} \cap \Lambda_{r} \boldsymbol{G}_{\mathrm{t}}^{\mathbb{C}}$ to denote the elements of $\Lambda \boldsymbol{G}_{\mathrm{t}}^{\mathbb{C}}$ whose entries also converge under the $\mathcal{W}_{r}$-topology, i.e., 
the "common part" of $\Lambda \boldsymbol{G}_{\mathrm{t}}^{\mathbb{C}}$ and $\Lambda_{r} \boldsymbol{G}_{\mathrm{t}}^{\mathbb{C}}$. An element $L \in \Lambda \boldsymbol{G}_{\mathrm{t}}^{\mathbb{C}} \cap \Lambda_{r} \boldsymbol{G}_{\mathrm{t}}^{\mathbb{C}}$ is said to be unitarizable via $r$-dressing if there is a $T_{+} \in \Lambda_{r}^{+} \boldsymbol{G}_{\mathrm{t}}^{\mathbb{C}}$ such that the element $T_{+} L T_{+}^{-1}$ of $\Lambda_{r} G_{\mathrm{t}}^{\mathbb{C}}$ is $r$-unitary, i.e., has a continuous extension up to $\mathbb{S}^{1}$ and is unitary on $\mathbb{S}^{1}$. In this case, if $0<r<1$, then $T_{+} L T_{+}^{-1}$ is actually holomorphic on $\{\lambda \in \mathbb{C} ; r<|\lambda|<1 / r\}$.

Sometimes, we will abbreviate unitarization via 1-dressing as unitarization via dressing.

The following proposition shows that all the elements of $\Lambda_{r} \boldsymbol{G}_{\mathrm{t}}^{\mathbb{C}}$ (not only just the elements of $\Lambda_{r}^{+} \boldsymbol{G}_{\mathrm{t}}^{\mathbb{C}}$ ) can be used for unitarization.

Proposition 2.1. Let $r \in(0,1]$. An element $L \in \Lambda \boldsymbol{G}_{\mathrm{t}}^{\mathbb{C}} \cap \Lambda_{r} \boldsymbol{G}_{\mathrm{t}}^{\mathbb{C}}$ is unitarizable via $r$-dressing if and only if there is a $T \in \Lambda_{r} \boldsymbol{G}_{\mathrm{t}}^{\mathbb{C}}$ such that the element $T L T^{-1}$ of $\Lambda_{r} \boldsymbol{G}_{\mathrm{t}}^{\mathbb{C}}$ is r-unitary.

Proof. The necessity is clear. To show the sufficiency, let $T=T_{\mathrm{u}} T_{+}$ be the $r$-Iwasawa splitting of $T$ with $T_{\mathrm{u}} \in \Lambda_{r} \boldsymbol{G}_{\mathrm{t}}$ and $T_{+} \in \Lambda_{r, \boldsymbol{B}}^{+} \boldsymbol{G}_{\mathrm{t}}^{\mathbb{C}}$, then $T_{+} L T_{+}^{-1}=T_{\mathrm{u}}^{-1} \cdot T L T^{-1} \cdot T_{\mathrm{u}}$ is $r$-unitary, and hence $L$ is unitarizable via $r$-dressing.

Conditions for unitarizability

The following proposition (see Theorem 3.5 in [3]) gives a characterization of an important class of unitarizable loop matrices. Recall that an element of $\operatorname{SL}(2, \mathbb{C})$ is semi-simple if and only if it is diagonalizable.

Proposition 2.2. i) Let $r \in(0,1]$. If $L \in \Lambda \boldsymbol{G}_{\mathrm{t}}^{\mathbb{C}} \cap \Lambda_{r} \boldsymbol{G}_{\mathrm{t}}^{\mathbb{C}}$ is unitarizable via $r$-dressing, then

$$
\operatorname{tr} L(\lambda) \in \mathbb{R} \text { and }|\operatorname{tr} L(\lambda)| \leq 2 \text { for all } \lambda \in \mathbb{S}^{1} .
$$

ii) Assume that $L \in \Lambda \boldsymbol{G}_{\mathrm{t}}^{\mathbb{C}}$ is holomorphic on a neighborhood of $\mathbb{S}^{1}$ and semi-simple on $\mathbb{S}^{1}$. If $L$ satisfies (2.3), then it is unitarizable via $r$-dressing for some $r \in(0,1]$.

Remark 2.4. Note that $L \in \Lambda \boldsymbol{G}_{\mathrm{t}}^{\mathbb{C}}$ satisfies (2.3) if and only if the eigenvalues of $L(\lambda)$ are unimodular for all $\lambda \in \mathbb{S}^{1}$.

In this paper, we will use $v(\lambda)$ to denote an eigenvalues of $L(\lambda)$, opposite to [3], in which $v(\lambda)$ is used to stand for the imaginary part of an eigenvalues. So, when $L(\lambda)$ satisfies $(2.3)$, its eigenvalues are $v(\lambda)$ and $1 / v(\lambda)$. 
Proposition 2.5. Assume that $L \in \Lambda \boldsymbol{G}_{\mathrm{t}}^{\mathbb{C}}$ is holomorphic on a neighborhood of $\mathbb{S}^{1}$ and satisfies (2.3). Then, the eigenvalues $v(\lambda)$ and $1 / v(\lambda)$ of $L(\lambda)$ can be chosen to depend holomorphically on $\lambda$ on a neighborhood of $\mathbb{S}^{1}$. The real parts of $v$ and $1 / v$ are both even in $\lambda$, and the imaginary parts of $v$ and $1 / v$ are either both even in $\lambda$ or both odd in $\lambda$.

Proof. Note that $4-(\operatorname{tr} L)^{2}$ is even in $\lambda$. From (2.3) we deduce that $4-(\operatorname{tr} L)^{2}$ is real on $\mathbb{S}^{1}$ and only has zeros of even order on $\mathbb{S}^{1}$. Thus, by Lemma 1.1, there is a function $g$ which is holomorphic on a neighborhood of $\mathbb{S}^{1}$ and satisfies $g^{2}=4-(\operatorname{tr} L)^{2}$. So, we can take $v=(\operatorname{tr} L+\mathrm{i} g) / 2$ and $1 / v=(\operatorname{tr} L-\mathrm{i} g) / 2$. The real parts both equal $\operatorname{tr} L / 2$ and are even in $\lambda$, while $g$ must be either even in $\lambda$ or odd in $\lambda$.

The eigenvalues $v(\lambda)$ and $1 / v(\lambda)$ of $L(\lambda)$ that depend holomorphically on $\lambda$ on a neighborhood of $\mathbb{S}^{1}$, as produced in Proposition 2.5 , will be called holomorphic eigenvalues. We will always use such eigenvalues. Moreover, when the imaginary parts of $v$ and $1 / v$ are even in $\lambda$, we will say that we have the even case; while when the imaginary parts of $v$ and $1 / v$ are odd in $\lambda$, the situation will called the odd case.

We note that the holomorphic eigenvalues of the monodromy of a noncylindrical Delaunay end are even in $\lambda$, while those of a cylindrical end are not. These examples motivate our distinction between the even case and odd case.

Using the concept of holomorphic eigenvalues, we have the following refinement of Part ii) of Proposition 2.2.

THEOREM 2.6. Let $L=\left(l_{i j}\right) \in \Lambda \boldsymbol{G}_{\mathrm{t}}^{\mathbb{C}}$ be holomorphic on a neighborhood of $\mathbb{S}^{1}$ and satisfy (2.3). Assume that the holomorphic eigenvalues $v$ and $1 / v$ of $L$ are not always equal on $\mathbb{S}^{1}$, i.e., $\operatorname{tr} L$ is not always equal to \pm 2 . Then, for each $r \in(0,1)$ sufficiently close to $1, L$ is unitarizable via $r$-dressing.

Proof. Our proof is basically the one of Theorem 3.5 in [3]. It is presented here because some details will be needed later.

In the even case, if we set

$$
T=\left(\begin{array}{cc}
1 & l_{12} /\left(v-l_{22}\right) \\
-l_{21} /(v-1 / v) & \left(l_{11}-1 / v\right) /(v-1 / v)
\end{array}\right)
$$


when $L$ is not triangular (i.e., $l_{12} \not \equiv 0 \not \equiv l_{21}$, which imply that neither of $l_{11}$ and $l_{22}$ is always equal to any of $v$ and $1 / v$ on $\mathbb{S}^{1}$ ),

$$
T=\left(\begin{array}{cc}
1 & 0 \\
l_{21} /(1 / v-v) & 1
\end{array}\right)
$$

when $L$ is lower triangular (i.e., $l_{12} \equiv 0$ ), and

$$
T=\left(\begin{array}{cc}
1 & l_{12} /(v-1 / v) \\
0 & 1
\end{array}\right)
$$

when $L$ is upper triangular (i.e., $\left.l_{21} \equiv 0\right)$, then for each $r \in(0,1)$ sufficiently close to $1, T \in \Lambda_{r} \boldsymbol{G}_{\mathrm{t}}^{\mathbb{C}}$ and it diagonalizes $L$ into $\Lambda_{r} \boldsymbol{G}_{\mathrm{t}}$, i.e.,

$$
T L T^{-1}=\left(\begin{array}{cc}
v & 0 \\
0 & 1 / v
\end{array}\right) \in \Lambda_{r} \boldsymbol{G}_{\mathrm{t}} .
$$

In the odd case, $l_{12} v_{\mathrm{I}} \not \equiv 0$ is even in $\lambda$, where $v=v_{\mathrm{R}}+\mathrm{i} v_{\mathrm{I}}$ with $v_{\mathrm{R}}$ and $v_{\text {I }}$ being real on $\mathbb{S}^{1}$. Moreover, for each $r \in(0,1)$ sufficiently close to 1 , we have that $T \in \Lambda_{r} G_{\mathrm{t}}^{\mathbb{C}}$ and

$$
T L T^{-1}=\left(\begin{array}{cc}
v_{\mathrm{R}} & \mathrm{i} v_{\mathrm{I}} \\
\mathrm{i} v_{\mathrm{I}} & v_{\mathrm{R}}
\end{array}\right) \in \Lambda_{r} \boldsymbol{G}_{\mathrm{t}},
$$

where

$$
T=\frac{\mathrm{i}}{\sqrt{\mathrm{i} l_{12} v_{\mathrm{I}}}}\left(\begin{array}{cc}
v_{\mathrm{R}}-l_{11} & -l_{12} \\
-\mathrm{i} v_{\mathrm{I}} & 0
\end{array}\right)
$$

if $\mathrm{i} l_{12} v_{\mathrm{I}}$ has an even root $\sqrt{\mathrm{i} l_{12} v_{\mathrm{I}}}$ which is holomorphic along $\mathbb{S}_{r}^{1}$, and

$$
T=\frac{1}{\sqrt{\mathrm{i} l_{12} v_{\mathrm{I}}}}\left(\begin{array}{cc}
\mathrm{i} v_{\mathrm{I}} & 0 \\
l_{11}-v_{\mathrm{R}} & l_{12}
\end{array}\right)
$$

if $\mathrm{i} l_{12} v_{\mathrm{I}}$ has an odd root $\sqrt{\mathrm{i} l_{12} v_{\mathrm{I}}}$ which is holomorphic along $\mathbb{S}_{r}^{1}$.

Remark 2.14. We emphasize that the matrices in (2.7)-(2.9) are meromorphic on a neighborhood of $\mathbb{S}^{1}$, while the matrices in (2.12) and (2.13) are holomorphic on $\mathcal{D} \backslash \overline{\mathcal{D}_{s}}$ for some $s \in(0,1)$ and the product of any two of their entries has a meromorphic extension to a neighborhood of $\mathbb{S}^{1}$. 
The form given in (2.11) for elements of $\Lambda_{r} \boldsymbol{G}_{\mathrm{t}}$ is called the anti-diagonal form, while the one in (2.10) the diagonal form. All the elements of $\Lambda_{r} \boldsymbol{G}_{\mathrm{t}}$ in the anti-diagonal form make up a subgroup of $\Lambda_{r} \boldsymbol{G}_{\mathrm{t}}$, to be denoted by $\Lambda_{r}^{\mathrm{a}} \boldsymbol{G}_{\mathrm{t}}$ (and sometimes abbreviated as $\Lambda^{\mathrm{a}} \boldsymbol{G}_{\mathrm{t}}$ when $r=1$ ). Similarly, we will use $\Lambda_{r}^{\mathrm{d}} \boldsymbol{G}_{\mathrm{t}}$ to denote the subgroup of $\Lambda_{r} \boldsymbol{G}_{\mathrm{t}}$ consisting of the diagonal elements of $\Lambda_{r} \boldsymbol{G}_{\mathrm{t}}$ (and frequently abbreviate $\Lambda_{1}^{\mathrm{d}} \boldsymbol{G}_{\mathrm{t}}$ as $\Lambda^{\mathrm{d}} \boldsymbol{G}_{\mathrm{t}}$ ).

Remark 2.15. Let $r \in(0,1]$.

i) If $L \in \Lambda_{r} \boldsymbol{G}_{\mathrm{t}}^{\mathbb{C}}$ is diagonalizable in $\Lambda_{r} \boldsymbol{G}_{\mathrm{t}}^{\mathbb{C}}$, then its eigenvalues are equal for all $\lambda \in \mathbb{S}_{r}^{1}$ if and only if $L \equiv I$ or $-I$.

ii) Assume that $L \in \Lambda_{r} \boldsymbol{G}_{\mathrm{t}}^{\mathbb{C}} \backslash\{ \pm I\}$ is diagonalized by $T \in \Lambda_{r} \boldsymbol{G}_{\mathrm{t}}^{\mathbb{C}}$, and the eigenvalues of $L$ are holomorphic on a neighborhood of $\mathbb{S}_{r}^{1}$. Then, the general $S \in \Lambda_{r} \boldsymbol{G}_{\mathrm{t}}^{\mathbb{C}}$ diagonalizing $L$ differs from $T$ by a diagonal or off-diagonal factor on the left. Note that a diagonal factor on the left does not change $T L T^{-1}$, while an off-diagonal one only interchanges the eigenvalues in $T L T^{-1}$.

General form of all unitarizing matrices

If the eigenvalues of a matrix $L \in \mathrm{SL}(2, \mathbb{C})$ are distinct and have unit modulus, then there is a matrix $T \in \mathrm{SL}(2, \mathbb{C})$ such that $T L T^{-1}$ is diagonal (and hence unitary), and the general $S \in \mathrm{SL}(2, \mathbb{C})$ unitarizing $L$ has the form $S=U D T$ with unitary $U$ and diagonal $D$. We generalize this matrix result to the loop group setting.

TheOREM 2.16. Let $L \in \Lambda \boldsymbol{G}_{\mathrm{t}}^{\mathbb{C}}$ be holomorphic on a neighborhood of $\mathbb{S}^{1}$.

i) If $T \in \Lambda \boldsymbol{G}_{\mathrm{t}}^{\mathbb{C}}$ diagonalizes $L$ into $\Lambda \boldsymbol{G}_{\mathrm{t}}$ and $L \notin\{ \pm I\}$, then the general $S \in \Lambda \boldsymbol{G}_{\mathrm{t}}^{\mathbb{C}}$ unitarizing $L$ has the form $S=U G_{+} T$ with $U \in \Lambda \boldsymbol{G}_{\mathrm{t}}$ and $\operatorname{diagonal} G_{+} \in \Lambda^{+} \boldsymbol{G}_{\mathrm{t}}^{\mathbb{C}}$.

ii) If $T \in \Lambda \boldsymbol{G}_{\mathrm{t}}^{\mathbb{C}}$ conjugates $L$ into $\Lambda^{\mathrm{a}} \boldsymbol{G}_{\mathrm{t}}$ and $L \notin\{ \pm I\}$, then the general $S \in \Lambda \boldsymbol{G}_{\mathrm{t}}^{\mathbb{C}}$ unitarizing $L$ has the form $S=U G_{+} T$ with $U \in \Lambda G_{\mathrm{t}}$ and

$$
G_{+}=\left(\begin{array}{cc}
\cosh \alpha_{+} & \sinh \alpha_{+} \\
\sinh \alpha_{+} & \cosh \alpha_{+}
\end{array}\right)
$$

for an odd $\alpha_{+} \in \mathcal{W}^{+}$.

iii) Assume that $L$ satisfies (2.3), and the two holomorphic eigenvalues of $L$ are even in $\lambda$ and not always equal to each other. Let $T$ be the matrix given by (2.7) or (2.8) or (2.9) for L. Then, for any $r \in(0,1)$ sufficiently 
close to 1 , the general $S \in \Lambda_{r} \boldsymbol{G}_{\mathrm{t}}^{\mathbb{C}}$ unitarizing $L$ has the form $S=U G_{+} T$ with $U \in \Lambda_{r} \boldsymbol{G}_{\mathrm{t}}$ and diagonal $G_{+} \in \Lambda_{r}^{+} \boldsymbol{G}_{\mathrm{t}}^{\mathbb{C}}$.

iv) Assume that $L$ satisfies (2.3), and the two holomorphic eigenvalues of $L$ are not even in $\lambda$. Let $T$ be the matrix given by (2.12) or (2.13) for $L$. Then, for any $r \in(0,1)$ sufficiently close to 1 , the general $S \in \Lambda_{r} \boldsymbol{G}_{t}^{\mathbb{C}}$ unitarizing $L$ has the form $S=U G_{+} T$ with $U \in \Lambda_{r} \boldsymbol{G}_{\mathrm{t}}$ and $G_{+} \in \Lambda_{r}^{+} \boldsymbol{G}_{\mathrm{t}}^{\mathbb{C}}$ given by (2.17) for an odd $\alpha_{+} \in \mathcal{W}_{r}^{+}$.

Proof. First, we show Parts i) and iii). We only need to prove the necessity of the form of $S$, since the sufficiency is trivial. Set

$$
T L T^{-1}=\left(\begin{array}{cc}
v & 0 \\
0 & 1 / v
\end{array}\right) .
$$

By our assumptions, $v-1 / v$ has at most finitely many zeros on $\mathbb{S}^{1}$. Note also that $1 / v=\bar{v}$ on $\mathbb{S}^{1}$.

i) Now, assume that $S \in \Lambda G_{\mathrm{t}}^{\mathbb{C}}$ unitarizes $L$. Let $S T^{-1}=U G_{+}$be the Iwasawa splitting of $S T^{-1}$ and set

$$
G_{+}=\left(\begin{array}{ll}
a & b \\
c & d
\end{array}\right)
$$

then

$$
\begin{aligned}
& \left(\begin{array}{cc}
v+b c(v-1 / v) & a b(1 / v-v) \\
c d(v-1 / v) & 1 / v+b c(1 / v-v)
\end{array}\right) \\
& \quad=G_{+}\left(\begin{array}{cc}
v & 0 \\
0 & 1 / v
\end{array}\right) G_{+}^{-1}=U^{-1} \cdot S L S^{-1} \cdot U
\end{aligned}
$$

is unitary, i.e.,

$$
b c(1 / v-v)=\overline{b c(v-1 / v)}, \quad c d(v-1 / v)=-\overline{a b(1 / v-v)} \quad \text { on } \mathbb{S}^{1} .
$$

The first identity of $(2.21)$ implies that $b c$ is real on $\mathbb{S}^{1}$. Hence, $b c$ has a holomorphic extension to the whole Riemann sphere $\mathbb{S}^{2}$, since it is holomorphic inside $\mathcal{D}$ (because $b, c \in \mathcal{W}^{+}$). So, $b c$ is constant and actually equals 0 , since $b(0) c(0)=0$. Thus, either $b=0$ or $c=0$. Moreover, $a d=a d-b c=1$. Therefore, the second identity of (2.21) yields that $b=c=0$, i.e., $G_{+}$is diagonal.

iii) By our assumptions, there is an $s \in(0,1)$ such that $L$ and its eigenvalues $v$ and $1 / v$ are holomorphic on $\mathcal{N}:=\mathcal{D}_{1 / s} \backslash \overline{\mathcal{D}_{s}}, v-1 / v$ does 
not have any zero on $\mathcal{N} \backslash \mathbb{S}^{1}$, and $T$ is holomorphic on $\mathcal{N} \backslash \mathbb{S}^{1}$. Fix an $r \in(s, 1)$. Note that this $r$ can be as close to 1 as one wants. Now, assume that $S \in \Lambda_{r} G_{\mathrm{t}}^{\mathbb{C}}$ unitarizes $L$. Let $S T^{-1}=U G_{+}$be the $r$-Iwasawa splitting of $S T^{-1}$ and define $a, b, c$ and $d$ by (2.19). Then, $U^{-1} \cdot S L S^{-1} \cdot U$ is given by (2.20) and is $r$-unitary. Thus, $a b, b c$ and $c d$ have meromorphic extensions to $\mathcal{D}_{1 / r}$, their poles in $\mathcal{D}_{1 / r}$ are on $\mathbb{S}^{1}$, and

$$
b c=\overline{b c}, a b=-\overline{c d} \quad \text { on } \mathbb{S}^{1}
$$

So, $a b, b c, c d$ and $a d=1+b c$ are meromorphic functions on the whole Riemann sphere $\mathbb{S}^{2}$ with poles only on $\mathbb{S}^{1}$, and $b c$ is real on $\mathbb{S}^{1}$. Since $a d \cdot b c=a b \cdot c d$ on $\mathcal{D}_{r}$ and hence on $\mathbb{S}^{2}$, from the second identity of $(2.22)$ we obtain that $a d \cdot b c=-a b \cdot \overline{a b}$ on $\mathbb{S}^{1}$, which together with $a d=1+b c$ yield that

$$
(1+b c) b c=-a b \cdot \overline{a b} \leq 0 \quad \text { on } \mathbb{S}^{1}
$$

The reality of $b c$ on $\mathbb{S}^{1}$ now implies that $-1 \leq b c \leq 0$ on $\mathbb{S}^{1}$. Thus, $b c$ does not have any pole on $\mathbb{S}^{1}$ and hence is actually holomorphic on the whole Riemann sphere $\mathbb{S}^{2}$. The rest of the arguments for this part are the same as the corresponding ones for Part i).

Next, we show Parts ii) and iv). We note that Parts i) and iii) are also true for the untwisted loop group (in this setting, $\boldsymbol{B}$ is replaced by the subgroup of $\operatorname{SL}(2, \mathbb{C})$ consisting of the upper triangular matrices with positive diagonal), since for any $G_{+}$given by (2.19) in the untwisted version of $\Lambda_{\boldsymbol{B}}^{+} \boldsymbol{G}_{\mathrm{t}}^{\mathbb{C}}$ or $\Lambda_{r, \boldsymbol{B}}^{+} \boldsymbol{G}_{\mathrm{t}}^{\mathbb{C}}$ we still have that $c(0)=0$.

ii) Let $S, U, G_{+}, a, b, c$ and $d$ be as in the proof of Part i). Set

$$
T_{*}=\frac{\mathrm{i}}{\sqrt{2}}\left(\begin{array}{cc}
1 & 1 \\
1 & -1
\end{array}\right),
$$

then $T_{*} \in \mathrm{SU}(2)$, and

$$
T_{*}\left(\begin{array}{ll}
v_{\mathrm{R}} & \mathrm{i} v_{\mathrm{I}} \\
\mathrm{i} v_{\mathrm{I}} & v_{\mathrm{R}}
\end{array}\right) T_{*}^{-1}=\left(\begin{array}{cc}
v & 0 \\
0 & 1 / v
\end{array}\right),
$$

which is only in the untwisted loop group in general. Note that $T_{*} T$ diagonalizes $L$. Split $G_{+}$into its constant term times its "strictly positive 
part":

$$
\begin{aligned}
G_{+}(\lambda) & =\left(\begin{array}{cc}
\mathrm{e}^{\alpha_{0}} & 0 \\
0 & \mathrm{e}^{-\alpha_{0}}
\end{array}\right) G_{++}(\lambda) \\
& =\left(\begin{array}{cc}
\mathrm{e}^{\alpha_{0}} & 0 \\
0 & \mathrm{e}^{-\alpha_{0}}
\end{array}\right)\left(I+G_{1} \lambda+G_{2} \lambda^{2}+\cdots\right)
\end{aligned}
$$

with $\alpha_{0} \in \mathbb{R}$. Then, the Iwasawa splitting of $S\left(T_{*} T\right)^{-1}=U G_{+} T_{*}^{-1}$ in the untwisted loop group is

$$
U T_{*}^{-1} \frac{1}{\beta}\left(\begin{array}{cc}
\cosh \alpha_{0} & -\sinh \alpha_{0} \\
\sinh \alpha_{0} & \cosh \alpha_{0}
\end{array}\right) \cdot\left(\begin{array}{cc}
\beta & \left(\beta+\frac{1}{\beta}\right) \tanh \alpha_{0} \\
0 & \frac{1}{\beta}
\end{array}\right) T_{*} G_{++} T_{*}^{-1},
$$

where $\beta=\sqrt{\cosh ^{2} \alpha_{0}+\sinh ^{2} \alpha_{0}}$. Thus,

$$
\left(\begin{array}{cc}
\beta & \left(\beta+\frac{1}{\beta}\right) \tanh \alpha_{0} \\
0 & \frac{1}{\beta}
\end{array}\right) T_{*} G_{++} T_{*}^{-1}
$$

is diagonal. So, $\alpha_{0}=0$, and $T_{*} G_{++} T_{*}^{-1}=\operatorname{diag}\left\{d_{++}, 1 / d_{++}\right\}$for some invertible $d_{++} \in \mathcal{W}^{+}$satisfying $d_{++}(0)=1$. Hence, $G_{+}$is given by $(2.17)$ with $\alpha_{+} \in \mathcal{W}^{+}$such that $\mathrm{e}^{\alpha_{+}}=d_{++}$. From $G_{+} \in \Lambda^{+} \boldsymbol{G}_{\mathrm{t}}^{\mathbb{C}}$ and the identity

$$
\left(\begin{array}{ll}
\cosh \gamma & \sinh \gamma \\
\sinh \gamma & \cosh \gamma
\end{array}\right)\left(\begin{array}{ll}
\cosh \delta & \sinh \delta \\
\sinh \delta & \cosh \delta
\end{array}\right)=\left(\begin{array}{cc}
\cosh (\gamma+\delta) & \sinh (\gamma+\delta) \\
\sinh (\gamma+\delta) & \cosh (\gamma+\delta)
\end{array}\right)
$$

we see that $\alpha_{+}$is odd.

iv) This part can be shown similar to Part ii).

Remark 2.30. In the odd case, i.e., in Parts ii) and iv), we just showed that

$$
S=U T_{*}^{-1} \cdot\left(\begin{array}{cc}
\mathrm{e}^{\alpha_{+}} & 0 \\
0 & \mathrm{e}^{-\alpha_{+}}
\end{array}\right) \cdot \widehat{T}
$$

with $U \in \Lambda_{r} \boldsymbol{G}_{\mathrm{t}}$, odd $\alpha_{+} \in \mathcal{W}_{r}^{+}$and $\widehat{T}=T_{*} T$, where $r=1$ for Part ii).

We note that in Theorem 2.16, whence $T$ is chosen, the factorization $S=U G_{+} T$ of $S$ with $U$ in $\Lambda \boldsymbol{G}_{\mathrm{t}}$ or $\Lambda_{r} \boldsymbol{G}_{\mathrm{t}}$ and $G_{+}$in $\Lambda_{\boldsymbol{B}}^{+} \boldsymbol{G}_{\mathrm{t}}^{\mathbb{C}}$ or $\Lambda_{r, \boldsymbol{B}}^{+} \boldsymbol{G}_{\mathrm{t}}^{\mathbb{C}}$ comes from the Iwasawa decomposition of $S T^{-1}$, and hence is unique. Moreover, Theorem 2.16 can be restated as the following: $G_{+}$either is diagonal or has the form given by (2.17), and this is the only condition on $S$. 
$\underline{\text { Lower and upper triangular loop matrices }}$

The following result gives a necessary and sufficient condition for unitarization via dressing for the lower triangular loop matrices. Note that now we are in the even case.

Proposition 2.32. Let $L \in \Lambda \boldsymbol{G}_{\mathrm{t}}^{\mathbb{C}}$ be holomorphic on a neighborhood of $\mathbb{S}^{1}$ and satisfy (2.3). Assume that $l_{12}=0$.

i) If $l_{11} \equiv l_{22}$, then $L$ is unitarizable via dressing if and only if $L= \pm I$.

ii) If $l_{11} \not \equiv l_{22}$, then $L$ is unitarizable via dressing if and only if the meromorphic function $l_{21} /\left(l_{11}-l_{22}\right)$ does not have any pole on $\mathbb{S}^{1}$, i.e., if and only if the vanishing order of $l_{21}$ at any $\lambda_{0} \in \mathbb{S}^{1}$ is at least that of $l_{11}-l_{22}$ at $\lambda_{0}$.

Proof. Since $l_{12}=0$, the holomorphic eigenvalues of $L$ are $l_{11}$ and $l_{22}$. The trace conditions imply that on $\mathbb{S}^{1}, l_{11}$ and $l_{22}$ are unimodular, and $l_{22}=1 / l_{11}=\overline{l_{11}}$.

i) From $l_{11} \equiv l_{22}$ we obtain that $l_{11} \equiv l_{22} \equiv \pm 1$ and hence the claim.

ii) Define $T$ by $(2.8)$ with $v=l_{11}$, then $T \in \Lambda_{r} \boldsymbol{G}_{\mathrm{t}}^{\mathbb{C}}$ for every real $r \neq 1$ sufficiently close to 1 , and

$$
T L T^{-1}=\left(\begin{array}{cc}
l_{11} & 0 \\
0 & l_{22}
\end{array}\right) \in \Lambda_{r} \boldsymbol{G}_{\mathrm{t}}
$$

for each real $r$ in a neighborhood of 1 .

If $l_{21} /\left(l_{11}-l_{22}\right)$ does not have any pole on $\mathbb{S}^{1}$, then it is holomorphic on a neighborhood of $\mathbb{S}^{1}$, and hence $T \in \Lambda \boldsymbol{G}_{\mathrm{t}}^{\mathbb{C}}$ unitarizes $L$.

Conversely, assume that $L$ is unitarizable via dressing, then there is a $G_{+} \in \Lambda^{+} \boldsymbol{G}_{\mathrm{t}}^{\mathbb{C}}$ such that $G_{+} L G_{+}^{-1} \in \Lambda \boldsymbol{G}_{\mathrm{t}}$. Let $r \in(0,1)$ be sufficiently close to 1 . Then, $G_{+} L G_{+}^{-1} \in \Lambda_{r} G_{\mathrm{t}}^{\mathbb{C}}, G_{+} L G_{+}^{-1}$ is hence $r$-unitary, and $G_{+}=$ $U D_{+} T$ for some $U \in \Lambda_{r} \boldsymbol{G}_{\mathrm{t}}$ and diagonal $D_{+} \in \Lambda_{r}^{+} \boldsymbol{G}_{\mathrm{t}}^{\mathbb{C}}$ by Theorem 2.16 Part iii). From $D_{+}=U^{-1} G_{+} T^{-1}$ we see that $D_{+}$has a continuous extension to $\overline{\mathcal{D}}$ with a finite number of points taken away that is holomorphic on $\mathcal{D}$. Set

$$
D_{+}=\left(\begin{array}{cc}
d_{+} & 0 \\
0 & 1 / d_{+}
\end{array}\right)
$$

then

$$
U^{-1} G_{+}=D_{+} T=\left(\begin{array}{cc}
d_{+} & 0 \\
l_{21} /\left[\left(l_{22}-l_{11}\right) d_{+}\right] & 1 / d_{+}
\end{array}\right) \in \Lambda \boldsymbol{G}_{\mathrm{t}}^{\mathbb{C}} .
$$


This implies that

$$
d_{+} \in \mathcal{W}^{*}, \quad l_{21} /\left[\left(l_{11}-l_{22}\right) d_{+}\right] \in \mathcal{W}
$$

As a consequence, $l_{21} /\left(l_{11}-l_{22}\right)$ is in $\mathcal{W}$ and hence does not have any pole on $\mathbb{S}^{1}$.

Note that the above proof also shows that in the case of Part ii) of the proposition, the general element of $\Lambda \boldsymbol{G}_{\mathrm{t}}$ unitarizing $L$ is of the form $U D_{+} T$, where $U \in \Lambda \boldsymbol{G}_{\mathrm{t}}, D_{+} \in \Lambda^{+} \boldsymbol{G}_{\mathrm{t}}^{\mathbb{C}}$ is diagonal, and $T$ is given by (2.8) with $v=l_{11}$.

Taking transpose inverse in the above proposition, one immediately obtains the corresponding result for upper triangular loop matrices.

Necessity of using $r$-dressing

Now, we present some loop matrices that can be unitarized via $r$ dressing with suitable $r<1$, but not via dressing. So, in general, $r$-dressing can not be replaced by dressing.

Example 2.37. For $s \in(0,+\infty) \backslash\{1 / 4,1 / 2\}$, let $t=1 / 2-s$ and

$$
f(\lambda)=2 \pi \sqrt{s^{2}+s t\left(\lambda^{-2}+\lambda^{2}\right)+t^{2}} .
$$

Since $s^{2}+s t\left(\lambda^{-2}+\lambda^{2}\right)+t^{2}$ is positive on $\mathbb{S}^{1}$ and even in $\lambda$, Lemma 1.1 implies that $f$ is holomorphic on a neighborhood of $\mathbb{S}^{1}$. Thus, by Proposition 2.32, for any odd function $g$ which is also holomorphic on a neighborhood of $\mathbb{S}^{1}$,

$$
L(\lambda)=\left(\begin{array}{cc}
\mathrm{e}^{\mathrm{i} f(\lambda)} & 0 \\
g(\lambda) & \mathrm{e}^{-\mathrm{i} f(\lambda)}
\end{array}\right) \in \Lambda \boldsymbol{G}_{\mathrm{t}}^{\mathbb{C}}
$$

is unitarizable via dressing if and only if \pm 1 are zeros of $g$ of order $\geq 2$. This is because \pm 1 are the only zeros of $2 \mathrm{i} \sin f=\mathrm{e}^{\mathrm{i} f}-\mathrm{e}^{-\mathrm{i} f}$, and they are double zeros. Note that $L$ is always unitarizable via $r$-dressing for each $r \in(0,1)$ sufficiently close to 1 .

In particular, the case with $g(\lambda)=\lambda$ yields

$$
\left(\begin{array}{cc}
\mathrm{e}^{\mathrm{i} f(\lambda)} & 0 \\
\lambda & \mathrm{e}^{-\mathrm{i} f(\lambda)}
\end{array}\right) \in \Lambda \boldsymbol{G}_{\mathrm{t}}^{\mathbb{C}},
$$

which is not unitarizable via dressing, but always unitarizable via $r$-dressing for each $r \in(0,1)$ sufficiently close to 1 . 


\section{$\S 3$. Simultaneous unitarization of two loop matrices}

In this section, we give conditions for the simultaneous unitarization of two loop matrices and show that under these conditions, there is a unique element of $\Lambda_{r, B}^{+} \boldsymbol{G}_{\mathrm{t}}^{\mathbb{C}}$ unitarizing them, unless the two loop matrices commute. As pointed out in the introduction, this uniqueness result will be used in Section 5 to deal with the closing conditions.

If one of the two loop matrices to be simultaneously unitarized is equal to $I$ or $-I$, then we only need to unitarize the other one. So, in the rest of this section, we will frequently exclude $\pm I$ from the two loop matrices to be simultaneously unitarized.

Commutativity of two loop matrices

First, we characterize the commutativity of two given loop matrices in terms of conditions on the loop matrices diagonalizing or anti-diagonalizing them individually.

Proposition 3.1. Let $0<r \leq 1$, and $L_{1}, L_{2} \in \Lambda_{r} \boldsymbol{G}_{\mathrm{t}}^{\mathbb{C}} \backslash\{ \pm I\}$. Assume that for $i=1$ and 2 , there exists a $T_{i} \in \Lambda_{r} \boldsymbol{G}_{\mathrm{t}}^{\mathbb{C}}$ such that $T_{i} L_{i} T_{i}^{-1}$ is in $\Lambda_{r}^{\mathrm{d}} \boldsymbol{G}_{\mathrm{t}} \cup \Lambda_{r}^{\mathrm{a}} \boldsymbol{G}_{\mathrm{t}}$ and holomorphic on a neighborhood of $\mathbb{S}_{r}^{1}$. Then, the following statements are equivalent:

i) $L_{1}$ and $L_{2}$ commute;

ii) $T_{1}$ conjugates $L_{1}$ and $L_{2}$ both into $\Lambda_{r}^{\mathrm{d}} \boldsymbol{G}_{\mathrm{t}}$ or both into $\Lambda_{r}^{\mathrm{a}} \boldsymbol{G}_{\mathrm{t}}$;

iii) $L_{1}$ and $L_{2}$ are both in the even case or both in the odd case, and $T_{*} T_{1} T_{2}^{-1} T_{*}^{-1}$ is either diagonal or off-diagonal, where $T_{*}=I$ in the even case and is given by (2.24) in the odd case.

Proof. Let $i=1$ or 2 . Set $\widehat{T}_{i}=T_{*} T_{i}$, where $T_{*}=I$ if $L_{i}$ is in the even case and $T_{*}$ is given by $(2.24)$ if $L_{i}$ is in the odd case. Then, $D_{i}:=\widehat{T}_{i} L_{i} \widehat{T}_{i}^{-1}$ is diagonal, and its diagonal entries are holomorphic on a neighborhood of $\mathbb{S}_{r}^{1}$ and not always equal. Thus, an $S$ in the untwisted $r$-loop group $\Lambda_{r} G^{\mathbb{C}}$ diagonalizes $L_{i}$ if and only if $S$ differs from $\widehat{T}_{i}$ by a diagonal or off-diagonal factor on the left, and an off-diagonal factor on the left just interchanges the diagonal entries of $\widehat{T}_{i} L_{i} \widehat{T}_{i}^{-1}$.

i) $\Longrightarrow$ ii). From $\widehat{T}_{1}^{-1} D_{1} \widehat{T}_{1} L_{2}=L_{1} L_{2}=L_{2} L_{1}=L_{2} \widehat{T}_{1}^{-1} D_{1} \widehat{T}_{1}$ we obtain

$$
D_{1} \cdot \widehat{T}_{1} L_{2} \widehat{T}_{1}^{-1}=\widehat{T}_{1} L_{2} \widehat{T}_{1}^{-1} \cdot D_{1},
$$


which implies that $\widehat{T}_{1} L_{2} \widehat{T}_{1}^{-1}$ is diagonal. Thus, if $L_{1}$ is in the even case, then $T_{1}$ conjugates $L_{1}$ and $L_{2}$ both into $\Lambda_{r}^{\mathrm{d}} \boldsymbol{G}_{\mathrm{t}}$; if $L_{1}$ is in the odd case, then $T_{1}$ conjugates $L_{1}$ and $L_{2}$ both into $\Lambda_{r}^{\mathrm{a}} \boldsymbol{G}_{\mathrm{t}}$.

ii) $\Longrightarrow \mathrm{i}$ ). This is trivial.

i) $\Longrightarrow$ iii). By Part ii), $L_{1}$ and $L_{2}$ are both in the even case or both in the odd case. Substituting $L_{2}=\widehat{T}_{2}^{-1} D_{2} \widehat{T}_{2}$ into (3.2) yields that

$$
D_{1} \cdot \widehat{T}_{1} \widehat{T}_{2}^{-1} D_{2} \widehat{T}_{2} \widehat{T}_{1}^{-1}=\widehat{T}_{1} \widehat{T}_{2}^{-1} D_{2} \widehat{T}_{2} \widehat{T}_{1}^{-1} \cdot D_{1},
$$

from which we see that $\widehat{T}_{1} \widehat{T}_{2}^{-1} D_{2} \widehat{T}_{2} \widehat{T}_{1}^{-1}$ is diagonal, and its entries are the same as those of $D_{2}$, but perhaps in different order. Thus, $T_{*} T_{1} T_{2}^{-1} T_{*}^{-1}=$ $\widehat{T}_{1} \widehat{T}_{2}^{-1}$ is either diagonal or off-diagonal.

iii) $\Longrightarrow$ i). Since now $\widehat{T}_{1} \widehat{T}_{2}^{-1} D_{2} \widehat{T}_{2} \widehat{T}_{1}^{-1}$ is diagonal by assumption, (3.3) follows, and hence

$$
\widehat{T}_{1}^{-1} D_{1} \widehat{T}_{1} \cdot \widehat{T}_{2}^{-1} D_{2} \widehat{T}_{2}=\widehat{T}_{2}^{-1} D_{2} \widehat{T}_{2} \cdot \widehat{T}_{1}^{-1} D_{1} \widehat{T}_{1},
$$

i.e., $L_{1} L_{2}=L_{2} L_{1}$.

Convention. Suggested by the above proof, whenever $T L T^{-1}$ is in $\Lambda_{r}^{\mathrm{d}} \boldsymbol{G}_{\mathrm{t}} \cup \Lambda_{r}^{\mathrm{a}} \boldsymbol{G}_{\mathrm{t}}$ and holomorphic on a neighborhood of $\mathbb{S}_{r}^{1}$ for some $r \in(0,1]$, we set $\widehat{T}=T_{*} T$, where $T_{*}=I$ if $L$ is in the even case and $T_{*}$ is given by (2.24) if $L$ is in the odd case.

$\underline{\text { Simultaneous unitarization of two loop matrices via dressing }}$

Now, we study the simultaneous unitarization of two loop matrices via dressing. It turns out that when the two loop matrices do not commute, there is at most one element of $\Lambda_{B}^{+} G_{\mathrm{t}}^{\mathbb{C}}$ simultaneously unitarizing them.

TheOREm 3.5. Let $L_{1}, L_{2} \in \Lambda \boldsymbol{G}_{\mathrm{t}}^{\mathbb{C}} \backslash\{ \pm I\}$ be holomorphic on a neighborhood of $\mathbb{S}^{1}$. Assume that for $i=1$ and 2 , there exists a $T_{i} \in \Lambda \boldsymbol{G}_{\mathrm{t}}^{\mathbb{C}}$ such that $T_{i} L_{i} T_{i}^{-1}$ is in $\Lambda^{\mathrm{d}} \boldsymbol{G}_{\mathrm{t}} \cup \Lambda^{\mathrm{a}} \boldsymbol{G}_{\mathrm{t}}$, and let $\widehat{T}_{i}$ be as in the above convention. Set

$$
\widehat{T}_{1} \widehat{T}_{2}^{-1}=\left(\begin{array}{ll}
a & b \\
c & d
\end{array}\right) .
$$

Then, $L_{1}$ and $L_{2}$ can be simultaneously unitarized via dressing if and only if either $b=c=0$, or $a=d=0$, or

(3.7) abcd $\not \equiv 0, \quad$ and $\frac{d}{\bar{a}},-\frac{c}{\bar{b}}$ are always finite, real and positive on $\mathbb{S}^{1}$. 
In the first two cases, $L_{1}$ and $L_{2}$ commute; while in the last case, there is a unique element of $\Lambda_{B}^{+} G_{\mathrm{t}}^{\mathbb{C}}$ simultaneously unitarizing $L_{1}$ and $L_{2}$.

Proof. By Proposition 3.1, $L_{1}$ and $L_{2}$ can be simultaneously conjugated into $\Lambda^{\mathrm{d}} \boldsymbol{G}_{\mathrm{t}}$ or into $\Lambda^{\mathrm{a}} \boldsymbol{G}_{\mathrm{t}}$ if and only if $\widehat{T}_{1} \widehat{T}_{2}^{-1}$ is either diagonal or off-diagonal, i.e., if and only if $L_{1}$ and $L_{2}$ commute. So, for the rest of this proof, we can assume that $\widehat{T}_{1} \widehat{T}_{2}^{-1}$ is neither diagonal nor off-diagonal.

For $i=1$ and 2 , let $T_{*, i}=I$ if $L_{i}$ is in the even case and $T_{*, i}$ equals the matrix in (2.24) if $L_{i}$ is in the odd case.

From Parts i) and ii) of Theorem 2.16 we know that $L_{1}$ and $L_{2}$ can be simultaneously unitarized via dressing using an $S \in \Lambda G_{\mathrm{t}}^{\mathbb{C}}$ if and only if there are $U_{1}, U_{2} \in \Lambda G$ and $G_{1}, G_{2} \in \Lambda_{B}^{+} G_{\mathrm{t}}^{\mathbb{C}}$ such that each of $G_{1}$ and $G_{2}$ is diagonal or has the form given in (2.17), and $S=U_{1} G_{1} T_{1}=U_{2} G_{2} T_{2}$. The latter is equivalent to $S=V_{1} K_{1} \widehat{T}_{1}=V_{2} K_{2} \widehat{T}_{2}$, where $V_{i}=U_{i} T_{*, i}^{-1}$ and $K_{i}=T_{*, i} G_{i} T_{*, i}^{-1}$ for $i=1$ and 2 . Note that $K_{1}$ and $K_{2}$ are diagonal by Remark 2.30, and that $V_{1}, V_{2}, K_{1}, K_{2}, \widehat{T}_{1}$ and $\widehat{T}_{2}$ are only in the untwisted loop group $\Lambda G^{\mathbb{C}}$ in general, and $V_{1}$ and $V_{2}$ are in $\Lambda G$.

Now, assume that $L_{1}$ and $L_{2}$ can be simultaneously unitarized via dressing. Then, using the notation of the last paragraph, $K_{1} \widehat{T}_{1} \widehat{T}_{2}^{-1} K_{2}^{-1}=$ $V_{1}^{-1} V_{2} \in \Lambda G^{\mathbb{C}}$ is unitary. Set

$$
K_{i}=\left(\begin{array}{cc}
k_{i} & 0 \\
0 & 1 / k_{i}
\end{array}\right) \quad \text { for } i=1 \text { and } 2 .
$$

Then,

$$
K_{1} \widehat{T}_{1} \widehat{T}_{2}^{-1} K_{2}^{-1}=\left(\begin{array}{cc}
a k_{1} / k_{2} & b k_{1} k_{2} \\
c /\left(k_{1} k_{2}\right) & d k_{2} / k_{1}
\end{array}\right)
$$

and hence

$$
d k_{2} / k_{1}=\overline{a k_{1} / k_{2}}, \quad c /\left(k_{1} k_{2}\right)=-\overline{b k_{1} k_{2}} \quad \text { on } \mathbb{S}^{1} .
$$

So, none of $a, b, c$ and $d$ is identically zero on $\mathbb{S}^{1}$ ( since $\widehat{T}_{1} \widehat{T}_{2}^{-1}$ is neither diagonal nor off-diagonal by our assumption), and $d / \bar{a}$ and $-c / \bar{b}$ are always finite, real and actually positive on $\mathbb{S}^{1}$. Thus, (3.7) is necessary.

Conversely, to show sufficiency, assume that (3.7) is true. By the Birkhoff decomposition on $\mathbb{S}^{1}$,

$$
\frac{d(\lambda)}{\overline{a(\lambda)}}=\frac{1}{m_{1}} \lambda^{m} f_{-}(\lambda) f_{+}(\lambda) \quad \text { on } \mathbb{S}^{1},
$$


where $m_{1}$ is a non-zero constant, $m \in \mathbb{Z}, f_{-} \in \mathcal{W}^{-}$never vanishes on $\overline{\mathcal{E}}:=\{|\lambda| \geq 1\} \cup\{\infty\}$ and satisfies $f_{-}(\infty)=1$, while $f_{+} \in \mathcal{W}^{+}$never vanishes on $\overline{\mathcal{D}}$ and satisfies $f_{+}(0)=1$. By the uniqueness of the Birkhoff decomposition, the reality of $d / \bar{a}$ on $\mathbb{S}^{1}$ is equivalent to that

$$
m_{1} \in \mathbb{R}, \quad m=0, \quad \overline{f_{+}(\lambda)}=f_{-}(\lambda) \quad \text { on } \mathbb{S}^{1} .
$$

Thus,

$$
\frac{d(\lambda)}{\overline{a(\lambda)}}=\frac{1}{m_{1}} \overline{f_{+}(\lambda)} f_{+}(\lambda) \text { on } \mathbb{S}^{1} .
$$

The positivity of $d / \bar{a}$ on $\mathbb{S}^{1}$ then implies that $m_{1}>0$. Similarly,

$$
-\frac{c(\lambda)}{\overline{b(\lambda)}}=n_{1} \overline{g_{+}(\lambda)} g_{+}(\lambda) \quad \text { on } \mathbb{S}^{1},
$$

where $n_{1}>0$, and $g_{+} \in \mathcal{W}^{+}$never vanishes on $\overline{\mathcal{D}}$ and satisfies $g_{+}(0)=1$. Set

$$
k_{1}=\left(n_{1} / m_{1}\right)^{1 / 4}\left(f_{+} g_{+}\right)^{1 / 2}, \quad k_{2}=\left(m_{1} n_{1}\right)^{1 / 4}\left(g_{+} / f_{+}\right)^{1 / 2} \quad \text { on } \overline{\mathcal{D}} \text {. }
$$

The roots here are possible since $f_{+}$and $g_{+}$can be represented in the form $\exp h_{+}$with $h_{+} \in \mathcal{W}_{+}$. Then, $k_{1}$ and $k_{2}$ belong to $\mathcal{W}^{+}$, never vanish on $\overline{\mathcal{D}}$, and satisfy (3.10). Note that $k_{1}(0)>0$ and $k_{2}(0)>0$. Define $K_{1}$ and $K_{2}$ by (3.8), then we have (3.9), and hence $V:=K_{1} \widehat{T}_{1} \widehat{T}_{2}^{-1} K_{2}^{-1} \in \Lambda G^{\mathbb{C}}$ is unitary. Moreover, from (3.10) or direct calculations we deduce that

$$
k_{1}^{2}{\bar{k}_{1}}^{2}=-\frac{c d}{\overline{a b}}, \quad k_{2}^{2}{\overline{k_{2}}}^{2}=-\frac{\bar{a} c}{\bar{b} d}=-\frac{\overline{a c}}{b d} \quad \text { on } \mathbb{S}^{1} .
$$

Next, we proceed in three non-overlapping situations separately.

If $L_{1}$ and $L_{2}$ are both in the even case, then $\widehat{T}_{1} \widehat{T}_{2}^{-1}=T_{1} T_{2}^{-1}$, which implies that $a$ and $d$ are even in $\lambda$, while $b$ and $c$ are odd in $\lambda$. Thus, $c d / \overline{a b}$ and $\overline{a c} /(b d)$ are even in $\lambda$. By (3.16) and Lemma 1.3, $k_{1}$ and $k_{2}$ are also even in $\lambda$. Hence, $G_{i}:=K_{i} \in \Lambda_{B}^{+} G_{\mathrm{t}}^{\mathbb{C}}$ for $i=1$ and 2 , and $U:=V=$ $K_{1} T_{1} T_{2}^{-1} K_{2}^{-1} \in \Lambda G_{\mathrm{t}}$. So, $L_{1}$ and $L_{2}$ can be simultaneously unitarized via dressing using $G_{1} T_{1}=U G_{2} T_{2}$.

If one of $L_{1}$ and $L_{2}$ is in the even case and the other is in the odd case, without loss of generality, we can assume that $L_{1}$ is in the even case and $L_{2}$ is in the odd case. Set

$$
T_{1} T_{2}^{-1}=\left(\begin{array}{cc}
\tilde{a} & \tilde{b} \\
\tilde{c} & \tilde{d}
\end{array}\right)
$$


Then, $\tilde{a}$ and $\tilde{d}$ are even in $\lambda, \tilde{b}$ and $\tilde{c}$ are odd in $\lambda$, and

$$
\frac{c d}{\overline{a b}}=\frac{\tilde{c}^{2}-\tilde{d}^{2}}{\overline{\tilde{a}^{2}-\tilde{b}^{2}}}, \quad \frac{\overline{a c}}{b d}=\frac{a c}{\overline{b d}}=\frac{\overline{(\tilde{a}+\tilde{b})(\tilde{c}+\tilde{d})}}{(\tilde{a}-\tilde{b})(\tilde{c}-\tilde{d})}=\frac{(\tilde{a}+\tilde{b})(\tilde{c}+\tilde{d})}{\overline{(\tilde{a}-\tilde{b})(\tilde{c}-\tilde{d})}} .
$$

So, $c d / \overline{a b}$ is even in $\lambda$, and

$$
\left(\frac{\overline{a c}}{b d}\right)(-\lambda)=\left(\frac{b d}{\overline{a c}}\right)(\lambda) .
$$

Thus, by (3.16) and Lemma 1.3, $k_{1}$ is even in $\lambda$, and $k_{2}=\mathrm{e}^{\alpha_{2}}$ for some odd $\alpha_{2} \in \mathcal{W}^{+}$. Then, we deduce that $G_{1}:=K_{1} \in \Lambda_{B}^{+} G_{\mathrm{t}}^{\mathbb{C}}, G_{2}:=\left(T_{*, 2}\right)^{-1} K_{2} T_{*, 2}$ $\in \Lambda_{B}^{+} G_{\mathrm{t}}^{\mathbb{C}}$, and

$$
U:=V T_{*, 2}=K_{1} \cdot T_{1} T_{2}^{-1} \cdot T_{*, 2}^{-1} K_{2}^{-1} T_{*, 2}=G_{1} \cdot T_{1} T_{2}^{-1} \cdot G_{2}^{-1} \in \Lambda G_{\mathrm{t}} .
$$

Thus, $G_{1} T_{1}=U G_{2} T_{2}$ simultaneously unitarizes $L_{1}$ and $L_{2}$.

If $L_{1}$ and $L_{2}$ are both in the odd case, similarly we can show that $k_{i}=\mathrm{e}^{\alpha_{i}}$ with an odd $\alpha_{i} \in \mathcal{W}^{+}$and $G_{i}:=T_{*, i}^{-1} K_{i} T_{*, i} \in \Lambda_{B}^{+} G_{\mathrm{t}}^{\mathbb{C}}$ for $i=1$ and 2 , and $U:=T_{*, 1}^{-1} V T_{*, 2}=G_{1} \cdot T_{1} T_{2}^{-1} \cdot G_{2}^{-1} \in \Lambda G_{\mathrm{t}}$. Hence, $L_{1}$ and $L_{2}$ can be simultaneously unitarized via dressing using $G_{1} T_{1}=U G_{2} T_{2}$.

Moreover, $k_{1}$ and $k_{2}$ are uniquely determined by $(3.16), k_{1}(0)>0$ and $k_{2}(0)>0$. Hence, in any case, $G_{1}$ and $G_{2}$ in $\Lambda_{B}^{+} G_{\mathrm{t}}^{\mathbb{C}}$ are also unique. Therefore, the unique element of $\Lambda_{B}^{+} G_{\mathrm{t}}^{\mathbb{C}}$ that simultaneously unitarizes $L_{1}$ and $L_{2}$ is the $\Lambda_{B}^{+} G_{\mathrm{t}}^{\mathbb{C}}$-part in the Iwasawa splitting of $G_{1} T_{1}$, i.e., that of $G_{2} T_{2}$.

Remark 3.21. In the case of the above theorem where $L_{1}$ and $L_{2}$ do not commute, up to a unitary factor on the left, the only element of $\Lambda G_{t}^{\mathbb{C}}$ simultaneously unitarizing $L_{1}$ and $L_{2}$ is

$$
G_{1} T_{1}=T_{*, 1}^{-1}\left(\begin{array}{cc}
\left(-\frac{c d}{\overline{a b}}\right)^{1 / 4} & 0 \\
0 & \left(-\frac{\overline{a b}}{c d}\right)^{1 / 4}
\end{array}\right) T_{*, 1} T_{1},
$$

where $T_{*, 1}$ is determined by $L_{1}$. The $\Lambda_{B}^{+} G_{\mathrm{t}}^{\mathbb{C}}$-part of the diagonal matrix in (3.22) is $K_{1}$ in the above proof. Such an element of $\Lambda \boldsymbol{G}_{\mathrm{t}}^{\mathbb{C}}$ can also be given by

$$
G_{2} T_{2}=T_{*, 2}^{-1}\left(\begin{array}{cc}
\left(-\frac{\overline{a c}}{b d}\right)^{1 / 4} & 0 \\
0 & \left(-\frac{b d}{\overline{a c}}\right)^{1 / 4}
\end{array}\right) T_{*, 2} T_{2},
$$

where $T_{*, 2}$ is determined by $L_{2}$. The $\Lambda_{B}^{+} G_{\mathrm{t}}^{\mathbb{C}}$-part of the diagonal matrix in (3.23) is $K_{2}$ in the above proof. 
Simultaneous unitarization of two loop matrices via $r$-dressing

Next, we deal with the simultaneous unitarization of two loop matrices via $r$-dressing with $r<1$ and, when they do not commute, find a unique element of $\Lambda_{r, \boldsymbol{B}}^{+} \boldsymbol{G}_{\mathrm{t}}^{\mathbb{C}}$ to do so.

TheOREM 3.24. Let $L_{1}, L_{2} \in \Lambda \boldsymbol{G}_{\mathrm{t}}^{\mathbb{C}}$ be holomorphic on a neighborhood of $\mathbb{S}^{1}$ and satisfy (2.3). Assume that for $i=1$ and 2 , the two holomorphic eigenvalues of $L_{i}$ are not always equal on $\mathbb{S}^{1}$. For $i=1$ and 2 , let $T_{i}$ be the matrix given by (2.7) or (2.8) or (2.9) or (2.12) or (2.13) for $L_{i}$, and define $\widehat{T}_{i}$ by the above convention. Moreover, set

$$
\left(\begin{array}{ll}
a & b \\
c & d
\end{array}\right)=\widehat{T}_{1} \widehat{T}_{2}^{-1}
$$

Then, $L_{1}$ and $L_{2}$ can be simultaneously unitarized via $r$-dressing for some $r \in(0,1)$ sufficiently close to 1 if and only if either $b=c=0$, or $a=d=0$, or as meromorphic functions on a neighborhood of $\mathbb{S}^{1}$,

abcd $\not \equiv 0$, and $\frac{d}{\bar{a}},-\frac{c}{\bar{b}}$ are real and non-negative on $\mathbb{S}^{1}$ (away from poles).

In the first two cases, $L_{1}$ and $L_{2}$ commute; while in the last case, there is a unique element of $\Lambda_{r, B}^{+} G_{\mathrm{t}}^{\mathbb{C}}$ simultaneously unitarizing $L_{1}$ and $L_{2}$, and we can choose $r$ such that it can be replaced by any number in $(r, 1)$.

Proof. Fix an $r \in(0,1)$ such that $L_{1}, L_{2}$ and their eigenvalues are holomorphic on $\mathcal{N}:=\mathcal{D}_{1 / s} \backslash \overline{\mathcal{D}_{s}}$ for some $s \in(0, r), T_{1}$ and $T_{2}$ are holomorphic on $\mathcal{N} \backslash \mathbb{S}^{1}$, and each of $a, b, c$ and $d$ is either identically zero or never zero on $\mathcal{N} \backslash \mathbb{S}^{1}$. Since this $r$ can be replaced by any number between $r$ and 1 , we only need to consider this $r$.

By Proposition 3.1, $L_{1}$ and $L_{2}$ can be simultaneously conjugated into $\Lambda_{r}^{\mathrm{d}} \boldsymbol{G}_{\mathrm{t}}$ or into $\Lambda_{r}^{\mathrm{a}} \boldsymbol{G}_{\mathrm{t}}$ if and only if $\widehat{T}_{1} \widehat{T}_{2}^{-1}$ is either diagonal or off-diagonal, i.e., if and only if $L_{1}$ and $L_{2}$ commute. So, for the rest of this proof, we can assume that $\widehat{T}_{1} \widehat{T}_{2}^{-1}$ is neither diagonal nor off-diagonal.

For $i=1$ and 2 , let $T_{*, i}=I$ if $L_{i}$ is in the even case and $T_{*, i}$ equals the matrix in (2.24) if $L_{i}$ is in the odd case.

By Parts iii) and iv) of Theorem 2.16, $L_{1}$ and $L_{2}$ can be simultaneously unitarized via $r$-dressing using an $S \in \Lambda_{r} \boldsymbol{G}_{\mathrm{t}}^{\mathbb{C}}$ if and only if there are $U_{1}, U_{2} \in$ $\Lambda_{r} \boldsymbol{G}_{\mathrm{t}}$ and $G_{1}, G_{2} \in \Lambda_{r, \boldsymbol{B}}^{+} \boldsymbol{G}_{\mathrm{t}}^{\mathbb{C}}$ such that each of $G_{1}$ and $G_{2}$ is diagonal or 
has the form given in (2.17), and $S=U_{1} G_{1} T_{1}=U_{2} G_{2} T_{2}$. The latter is equivalent to $S=V_{1} K_{1} \widehat{T}_{1}=V_{2} K_{2} \widehat{T}_{2}$, where $V_{i}=U_{i}\left(T_{*, i}\right)^{-1}$ and $K_{i}=$ $T_{*, i} G_{i}\left(T_{*, i}\right)^{-1}$ for $i=1$ and 2 . Note that $K_{1}$ and $K_{2}$ are diagonal by Remark 2.30, and that $V_{1}, V_{2}, K_{1}, K_{2}, \widehat{T}_{1}$ and $\widehat{T}_{2}$ are only in the untwisted $r$-loop group $\Lambda_{r} \boldsymbol{G}^{\mathbb{C}}$ in general, and $V_{1}$ and $V_{2}$ are in $\Lambda_{r} \boldsymbol{G}$.

Now, assume that $L_{1}$ and $L_{2}$ can be simultaneously unitarized via $r$ dressing. Then, using the notation of the last paragraph, $K_{1} \widehat{T}_{1} \widehat{T}_{2}^{-1} K_{2}^{-1}=$ $V_{1}^{-1} V_{2} \in \Lambda_{r} G^{\mathbb{C}}$ is $r$-unitary. Define $k_{1}$ and $k_{2}$ by (3.8), then (3.9) holds. Thus, the entries of $K_{1} \widehat{T}_{1} \widehat{T}_{2}^{-1} K_{2}^{-1}$ have holomorphic extensions to $\mathcal{D}_{1 / r} \backslash \overline{\mathcal{D}_{r}}$ and extend continuously to $\mathbb{S}_{1 / r}$, and for $\lambda \in \overline{\mathcal{D}_{1 / r}} \backslash \mathcal{D}_{r}$,

$$
\left(d k_{2} / k_{1}\right)(\lambda)=\overline{\left(a k_{1} / k_{2}\right)(1 / \bar{\lambda})}, \quad\left(c /\left(k_{1} k_{2}\right)\right)(\lambda)=-\overline{\left(b k_{1} k_{2}\right)(1 / \bar{\lambda})} .
$$

So, $a b c d \not \equiv 0$ on $\mathbb{S}^{1}, k_{1} k_{2}$ and $k_{1} / k_{2}$ have meromorphic extensions to $\mathcal{D}_{1 / r} \backslash \overline{\mathcal{D}_{r}}$ and extend continuously to $\mathbb{S}_{1 / r}$, and for $\lambda \in \overline{\mathcal{D}_{1 / r}} \backslash \mathcal{D}_{r}$,

$$
\left(\frac{k_{1}}{k_{2}}\right)(\lambda) \cdot \overline{\left(\frac{k_{1}}{k_{2}}\right)(1 / \bar{\lambda})}=\frac{d(\lambda)}{\overline{a(1 / \bar{\lambda})}}, \quad\left(k_{1} k_{2}\right)(\lambda) \cdot \overline{\left(k_{1} k_{2}\right)(1 / \bar{\lambda})}=-\frac{c(\lambda)}{\overline{b(1 / \bar{\lambda})}}
$$

On $\mathbb{S}^{1}$ (with a finite number of points taken away), (3.28) can be rewritten as

$$
\left(\frac{k_{1}}{k_{2}}\right) \cdot \overline{\left(\frac{k_{1}}{k_{2}}\right)}=\frac{d}{\bar{a}}, \quad k_{1} k_{2} \cdot \overline{k_{1} k_{2}}=-\frac{c}{\bar{b}},
$$

and the expressions in (3.28) are the unique meromorphic extensions of the ones here. By (3.29), as meromorphic functions, $d / \bar{a}$ and $-c / \bar{b}$ are real and non-negative on $\mathbb{S}^{1}$. Thus, (3.26) is necessary.

Conversely, to show sufficiency, assume that (3.26) is true. Then, each zero or pole of $d(\lambda) \overline{a(1 / \bar{\lambda})}$ on $\mathbb{S}^{1}$ has an even order. So,

$$
\frac{d(\lambda)}{\overline{a(1 / \bar{\lambda})}}=f(\lambda) \frac{\prod_{i=1}^{m_{2}}\left(1-\lambda / \lambda_{i}^{+}\right)\left(1-\lambda_{i}^{+} / \lambda\right)}{\prod_{j=1}^{m_{3}}\left(1-\lambda / \lambda_{j}^{-}\right)\left(1-\lambda_{j}^{-} / \lambda\right)} \quad \text { on } \mathcal{N}
$$

where $m_{2}, m_{3} \in \mathbb{N} \cup\{0\}, \lambda_{1}^{+}, \lambda_{2}^{+}, \ldots, \lambda_{m_{2}}^{+}, \lambda_{1}^{-}, \lambda_{2}^{-}, \ldots, \lambda_{m_{3}}^{-} \in \mathbb{S}^{1}$ (it is possible that some of them repeat) such that $\lambda_{i}^{+} \neq \lambda_{j}^{-}$for any $i$ and $j$, and $f$ is holomorphic and never vanishes on $\mathcal{N}$. By the Birkhoff decomposition on $\mathbb{S}^{1}$

$$
f(\lambda)=\frac{1}{m_{1}} \lambda^{m} f_{-}(\lambda) f_{+}(\lambda) \quad \text { on } \mathcal{N}
$$


where $m_{1}$ is a non-zero constant, $m \in \mathbb{Z}, f_{-} \in \mathcal{W}_{-}$never vanishes on $\overline{\mathcal{E}}=\{|\lambda| \geq 1\} \cup\{\infty\}$ and satisfies $f_{-}(\infty)=1$, while $f_{+} \in \mathcal{W}_{+}$never vanishes on $\overline{\mathcal{D}}$ and satisfies $f_{+}(0)=1$. The reality of $d(\lambda) / \overline{a(1 / \bar{\lambda})}$ on $\mathbb{S}^{1}$ is equivalent to that

$$
m_{1} \in \mathbb{R}, \quad m=0, \quad \overline{f_{+}(1 / \bar{\lambda})}=f_{-}(\lambda) \text { on } \mathcal{N} .
$$

In total, we have that

$$
\frac{d(\lambda)}{\overline{a(1 / \bar{\lambda})}}=\frac{1}{m_{1}} \overline{f_{+}(1 / \bar{\lambda})} f_{+}(\lambda) \frac{\prod_{i=1}^{m_{2}}\left(1-\lambda / \lambda_{i}^{+}\right)\left(1-\lambda_{i}^{+} / \lambda\right)}{\prod_{j=1}^{m_{3}}\left(1-\lambda / \lambda_{j}^{-}\right)\left(1-\lambda_{j}^{-} / \lambda\right)} \quad \text { on } \mathcal{N} \text {. }
$$

Since $d / \bar{a}$ is non-negative on $\mathbb{S}^{1}$, we obtain that $m_{1}>0$. Similarly,

$$
-\frac{c(\lambda)}{\overline{b(1 / \bar{\lambda})}}=n_{1} \overline{g_{+}(1 / \bar{\lambda})} g_{+}(\lambda) \frac{\prod_{i=1}^{n_{2}}\left(1-\lambda / \nu_{i}^{+}\right)\left(1-\nu_{i}^{+} / \lambda\right)}{\prod_{j=1}^{n_{3}}\left(1-\lambda / \nu_{j}^{-}\right)\left(1-\nu_{j}^{-} / \lambda\right)} \quad \text { on } \mathcal{N}
$$

where $n_{1}>0, n_{2}, n_{3} \in \mathbb{N} \cup\{0\}, \nu_{1}^{+}, \nu_{2}^{+}, \ldots, \nu_{n_{2}}^{+}, \nu_{1}^{-}, \nu_{2}^{-}, \ldots, \nu_{n_{3}}^{-} \in \mathbb{S}^{1}$ such that $\nu_{i}^{+} \neq \nu_{j}^{-}$for any $i$ and $j$, and $g_{+}$is a never-vanishing holomorphic function on $\mathcal{D}_{1 / s}$ satisfying $g_{+}(0)=1$. Set

$$
k_{1}(\lambda)=\left(\frac{n_{1}}{m_{1}}\right)^{1 / 4}\left[\frac{f_{+}(\lambda) g_{+}(\lambda) \prod_{i=1}^{m_{2}}\left(1-\lambda / \lambda_{i}^{+}\right) \prod_{k=1}^{n_{2}}\left(1-\lambda / \nu_{k}^{+}\right)}{\prod_{j=1}^{m_{3}}\left(1-\lambda / \lambda_{j}^{-}\right) \prod_{l=1}^{n_{3}}\left(1-\lambda / \nu_{l}^{-}\right)}\right]^{1 / 2},
$$

$$
k_{2}(\lambda)=\left(m_{1} n_{1}\right)^{1 / 4}\left[\frac{g_{+}(\lambda) \prod_{j=1}^{m_{3}}\left(1-\lambda / \lambda_{j}^{-}\right) \prod_{k=1}^{n_{2}}\left(1-\lambda / \nu_{k}^{+}\right)}{f_{+}(\lambda) \prod_{i=1}^{m_{2}}\left(1-\lambda / \lambda_{i}^{+}\right) \prod_{l=1}^{n_{3}}\left(1-\lambda / \nu_{l}^{-}\right)}\right]^{1 / 2}
$$

on $\mathcal{D}$. Then, $k_{1}$ and $k_{2}$ belong to $\mathcal{W}_{r}^{+}$, never vanish on $\mathcal{D}$, have continuous extensions to $\mathbb{S}^{1}$ with at most a finite number of points taken away, and satisfy $(3.28)$ on $\mathcal{D} \backslash \mathcal{D}_{r}$. Thus, we have $(3.27)$ on $\mathcal{D} \backslash \mathcal{D}_{r}$. Note that $k_{1}(0)>0$ and $k_{2}(0)>0$. Define $K_{1}$ and $K_{2}$ by (3.8), and consider $V:=K_{1} \widehat{T}_{1} \widehat{T}_{2}^{-1} K_{2}^{-1} \in \Lambda_{r} G^{\mathbb{C}}$. Since only $k_{1} k_{2}$ and $k_{1} / k_{2}$ occur in $V$, it is meromorphic on $\mathcal{N}$. By (3.27), $V$ is unitary and hence stays uniformly bounded at its continuous points on $\mathbb{S}^{1}$. Thus, the finitely many singularities of $V$ on $\mathbb{S}^{1}$ are removable. Therefore, $V$ is $r$-unitary. Moreover, (3.28) then holds on $\mathcal{N}$, and hence

$$
k_{1}^{2}(\lambda) \overline{k_{1}^{2}(1 / \bar{\lambda})}=-\frac{(c d)(\lambda)}{\overline{(a b)(1 / \bar{\lambda})}}, \quad k_{2}^{2}(\lambda) \overline{k_{2}^{2}(1 / \bar{\lambda})}=-\frac{\overline{(a c)(1 / \bar{\lambda})}}{(b d)(\lambda)} \quad \text { on } \mathcal{N} \text {. }
$$


Next, we proceed in three non-overlapping situations separately. Since the remaining arguments are similar to the corresponding ones in the proof of Theorem 3.5, we will only sketch them.

If $L_{1}$ and $L_{2}$ are both in the even case, then $c d / \overline{a b}$ and $\overline{a c} /(b d)$ are even in $\lambda$. By (3.37) and Lemma 1.3, $k_{1}$ and $k_{2}$ are also even in $\lambda$. Hence, $G_{i}:=K_{i} \in \Lambda_{r, \boldsymbol{B}}^{+} \boldsymbol{G}_{\mathrm{t}}^{\mathbb{C}}$ for $i=1$ and 2 , and $U:=V=K_{1} T_{1} T_{2}^{-1} K_{2}^{-1} \in \Lambda_{r} \boldsymbol{G}_{\mathrm{t}}$. So, $L_{1}$ and $L_{2}$ can be simultaneously unitarized via $r$-dressing using $G_{1} T_{1}=$ $U G_{2} T_{2}$.

If one $L_{1}$ and $L_{2}$ is in the even case and the other is in the odd case, without loss of generality, we can assume that $L_{1}$ is in the even case and $L_{2}$ is in the odd case. So, $c d / \overline{a b}$ is even in $\lambda$, and

$$
\left(\frac{\overline{a c}}{b d}\right)(-\lambda)=\left(\frac{b d}{\overline{a c}}\right)(\lambda) \text { on } \mathbb{S}^{1} .
$$

Thus, by (3.37) and Lemma 1.3, $k_{1}$ is even in $\lambda$, and $k_{2}=\mathrm{e}^{\alpha_{2}}$ for some odd $\alpha_{2} \in \mathcal{W}^{+} \subset \mathcal{W}_{r}^{+}$. Then, we deduce that $G_{1}:=K_{1} \in \Lambda_{r, B}^{+} G_{\mathrm{t}}^{\mathbb{C}}, G_{2}:=$ $\left(T_{*, 2}\right)^{-1} K_{2} T_{*, 2} \in \Lambda_{r, B}^{+} \boldsymbol{G}_{\mathrm{t}}^{\mathbb{C}}$, and $U:=V T_{*, 2}=G_{1} \cdot T_{1} T_{2}^{-1} \cdot G_{2}^{-1} \in \Lambda_{r} \boldsymbol{G}_{\mathrm{t}}$. Thus, $G_{1} T_{1}=U G_{2} T_{2}$ simultaneously unitarizes $L_{1}$ and $L_{2}$.

If $L_{1}$ and $L_{2}$ are both in the odd case, then $G_{i}:=T_{*, i}^{-1} K_{i} T_{*, i} \in \Lambda_{r, \boldsymbol{B}}^{+} \boldsymbol{G}_{\mathrm{t}}^{\mathbb{C}}$ for $i=1$ and 2 , and $U:=T_{*, 1}^{-1} V T_{*, 2}=G_{1} \cdot T_{1} T_{2}^{-1} \cdot G_{2}^{-1} \in \Lambda_{r} G_{\mathrm{t}}$. Hence, $L_{1}$ and $L_{2}$ can be simultaneously unitarized via $r$-dressing using $G_{1} T_{1}=$ $U G_{2} T_{2}$.

Moreover, $k_{1}$ and $k_{2}$ are uniquely determined by $(3.37), k_{1}(0)>0$ and $k_{2}(0)>0$. Hence, in any case, $G_{1}$ and $G_{2}$ in $\Lambda_{r, \boldsymbol{B}}^{+} \boldsymbol{G}_{\mathrm{t}}^{\mathbb{C}}$ are also unique. Therefore, the unique element of $\Lambda_{r, B}^{+} G_{\mathrm{t}}^{\mathbb{C}}$ that simultaneously unitarizes $L_{1}$ and $L_{2}$ is the $\Lambda_{r, B}^{+} G_{\mathrm{t}}^{\mathbb{C}}$-part in the Iwasawa splitting of $G_{1} T_{1}$, i.e., that of $G_{2} T_{2}$.

Remark 3.39. In the case of the above theorem where $L_{1}$ and $L_{2}$ do not commute, up to a unitary factor on the left, the meromorphic extension to $\mathbb{S}^{1}$ of the only element of $\Lambda_{r} \boldsymbol{G}_{\mathrm{t}}^{\mathbb{C}}$ simultaneously unitarizing $L_{1}$ and $L_{2}$ is given by (3.22) with $T_{*, 1}$ determined by $L_{1}$. The $\Lambda_{r, \boldsymbol{B}}^{+} \boldsymbol{G}_{\mathrm{t}}^{\mathbb{C}}$-part of the diagonal matrix in (3.22) is $K_{1}$ in the above proof. The meromorphic extension to $\mathbb{S}^{1}$ of such an element of $\Lambda_{r} \boldsymbol{G}_{\mathrm{t}}^{\mathbb{C}}$ can also be given by (3.23) with $T_{*, 2}$ determined by $L_{2}$. The $\Lambda_{r, \boldsymbol{B}}^{+} \boldsymbol{G}_{\mathrm{t}}^{\mathbb{C}}$-part of the diagonal matrix in (3.23) is $K_{2}$ in the above proof. 
Remark 3.40. We note that our above proof of Theorem 3.24 actually shows the following fact: when $L_{1}$ is diagonal or anti-diagonal, there exists an $r \in(0,1)$, as close to 1 as one wants, such that $\Lambda_{r}^{+} \boldsymbol{G}_{\mathrm{t}}^{\mathbb{C}}$ contains an element $S$ which commutes with $L_{1}$ and unitarizes $L_{2}$, i.e.,

$$
S L_{1} S^{-1}=L_{1}, \quad S L_{2} S^{-1} \text { is } r \text {-unitary. }
$$

In fact, if $L_{1}$ is diagonal or anti-diagonal, then $T_{1}=I$ and we can take $S=T_{*}^{-1} K_{1} T_{*}$, where $T_{*}$ is determined by $L_{1}$. A similar statement is true when $L_{1}$ is an $s$-unitary conjugate of its diagonal or anti-diagonal form for some $s \in[r, 1)$.

Remark 3.42. From (3.35) and (3.36) we see that in general, the $K_{1}$ and $K_{2}$ produced in the above proof can not be meromorphically extended to the whole $\mathbb{S}^{1}$. Thus, in general, the loop matrix simultaneously unitarizing $L_{1}$ and $L_{2}$ is not meromorphic on any neighborhood of $\mathbb{S}^{1}$.

Some examples

Now, we apply Theorems 3.5 and 3.24 to several special subcases. We start with the subcase where the two loop matrices to be simultaneously unitarized are both lower triangular, while the subcase where the two loop matrices are both upper triangular can be treated very similarly. Note that now we are in the even case in the sense of Section 2.

Corollary 3.43. Consider

$$
L_{1}=\left(\begin{array}{cc}
\mathrm{e}^{\mathrm{i} f} & 0 \\
p & \mathrm{e}^{-\mathrm{i} f}
\end{array}\right), \quad L_{2}=\left(\begin{array}{cc}
\mathrm{e}^{\mathrm{i} g} & 0 \\
q & \mathrm{e}^{-\mathrm{i} g}
\end{array}\right)
$$

in $\Lambda \boldsymbol{G}_{\mathrm{t}}^{\mathbb{C}}$ with real-valued $f$ and $g$ in $\mathcal{W}$. Assume that $f, g, p$ and $q$ are holomorphic on a neighborhood of $\mathbb{S}^{1}$, and none of $\sin f$ and $\sin g$ is identically 0 on $\mathbb{S}^{1}$.

i) The matrices $L_{1}$ and $L_{2}$ can be simultaneously unitarized via dressing if and only if $p / \sin f$ and $q / \sin g$ are always finite on $\mathbb{S}^{1}$ and $L_{1}$ and $L_{2}$ commute, if and only if

$$
\frac{p}{\sin f} \text { and } \frac{q}{\sin g} \text { are always finite and equal on } \mathbb{S}^{1} \text {. }
$$

In particular, if $p=0$ and $q \neq 0$ in $\mathcal{W}$, then $L_{1}$ and $L_{2}$ can not be simultaneously unitarized via dressing. 
ii) The matrices $L_{1}$ and $L_{2}$ can be simultaneously unitarized via $r$ dressing for some $r \in(0,1)$ sufficiently close to 1 if and only if they commute, i.e., if and only if

$$
\frac{p}{\sin f}=\frac{q}{\sin g} \text { on } \mathbb{S}^{1}
$$

as meromorphic functions. In this case, there is a unique element of $\Lambda_{r, \boldsymbol{B}}^{+} \boldsymbol{G}_{\mathrm{t}}^{\mathbb{C}}$ simultaneously unitarizing $L_{1}$ and $L_{2}$, and we can choose $r$ such that it can be replaced by any number in $(r, 1)$. In particular, if furthermore $p=0$ and $q \neq 0$ in $\mathcal{W}$, then $L_{1}$ and $L_{2}$ can not be simultaneously unitarized via $r$-dressing for any $r \in(0,1)$ sufficiently close to 1 .

Proof. Our assumptions and Proposition 2.32 imply that if $L_{1}$ and $L_{2}$ are unitarizable via dressing, then $p / \sin f$ and $q / \sin g$ are always finite on $\mathbb{S}^{1}$. So, following the proof of Theorem 2.6 , in this subcase we can take

$$
T_{1}=\left(\begin{array}{cc}
1 & 0 \\
\frac{\mathrm{i} p}{2 \sin f} & 1
\end{array}\right), \quad T_{2}=\left(\begin{array}{cc}
1 & 0 \\
\frac{\mathrm{i} q}{2 \sin g} & 1
\end{array}\right)
$$

Then,

$$
T_{1} T_{2}^{-1}=\left(\begin{array}{ccc}
1 & 0 \\
\frac{\mathrm{i} p}{2 \sin f}-\frac{\mathrm{i} q}{2 \sin g} & 1
\end{array}\right)
$$

Therefore, our results follow directly from Theorems 3.5 and 3.24.

Next, we examine the subcase where one of the two loop matrices is lower triangular and the other one is upper triangular. This subcase is also motivated by some situation in the construction of constant mean curvature trinoids. Note that we are again in the even case.

Corollary 3.49. Consider

$$
L_{1}=\left(\begin{array}{cc}
\mathrm{e}^{\mathrm{i} f} & 0 \\
p & \mathrm{e}^{-\mathrm{i} f}
\end{array}\right), \quad L_{2}=\left(\begin{array}{cc}
\mathrm{e}^{-\mathrm{i} g} & q \\
0 & \mathrm{e}^{\mathrm{i} g}
\end{array}\right)
$$

in $\Lambda \boldsymbol{G}_{\mathrm{t}}^{\mathbb{C}}$ with real-valued $f$ and $g$ in $\mathcal{W}$. Assume that $f, g, p$ and $q$ are holomorphic on a neighborhood of $\mathbb{S}^{1}$, and none of $\sin f, \sin g, p$ and $q$ is identically 0 on $\mathbb{S}^{1}$. 
i) The matrices $L_{1}$ and $L_{2}$ can be simultaneously unitarized via dressing if and only if

$$
\frac{p}{\sin f}, \frac{q}{\sin g} \text { are always finite, } p q \text { is real, }-4<\frac{p q}{\sin f \sin g}<0 \text { on } \mathbb{S}^{1} \text {. }
$$

In this case, there is a unique element of $\Lambda_{\boldsymbol{B}}^{+} \boldsymbol{G}_{\mathrm{t}}^{\mathbb{C}}$ simultaneously unitarizing $L_{1}$ and $L_{2}$.

ii) The matrices $L_{1}$ and $L_{2}$ can be simultaneously unitarized via $r$ dressing for some $r \in(0,1)$ sufficiently close to 1 if and only if

$$
4 \sin f \sin g+p q \not \equiv 0, \quad p q \text { is real, }-4 \leq \frac{p q}{\sin f \sin g} \leq 0 \text { on } \mathbb{S}^{1}
$$

as meromorphic functions. In this case, there is a unique element of $\Lambda_{r, \boldsymbol{B}}^{+} \boldsymbol{G}_{\mathrm{t}}^{\mathbb{C}}$ simultaneously unitarizing $L_{1}$ and $L_{2}$, and we can choose $r$ such that it can be replaced by any number in $(r, 1)$.

Note that the inequalities in (3.52) imply, for example, that the zeros of $\sin f \sin g$ on $\mathbb{S}^{1}$ are among the ones of $p q$ on $\mathbb{S}^{1}$.

Proof. i) By our assumptions and Proposition 2.32, if $L_{1}$ and $L_{2}$ are unitarizable via dressing, then $p / \sin f$ and $q / \sin g$ are always finite on $\mathbb{S}^{1}$. So, following the proof of Theorem 2.6, in this subcase we can take

$$
T_{1}=\left(\begin{array}{cc}
1 & 0 \\
\frac{\mathrm{i} p}{2 \sin f} & 1
\end{array}\right), \quad T_{2}=\left(\begin{array}{cc}
1 & \frac{\mathrm{i} q}{2 \sin g} \\
0 & 1
\end{array}\right)
$$

Then,

$$
T_{1} T_{2}^{-1}=\left(\begin{array}{cc}
1 & -\frac{\mathrm{i} q}{2 \sin g} \\
\frac{\mathrm{i} p}{2 \sin f} & \frac{p q}{4 \sin f \sin g}+1
\end{array}\right) .
$$

Thus, by Theorem $3.5, L_{1}$ and $L_{2}$ can be simultaneously unitarized via dressing if and only if

$\frac{p}{\sin f}, \frac{q}{\sin g}$ are always finite, $-\frac{p \sin g}{\bar{q} \sin f}, \frac{p q}{4 \sin f \sin g}+1 \in(0,+\infty)$ on $\mathbb{S}^{1}$.

Assume that $p / \sin f$ and $q / \sin g$ are always finite on $\mathbb{S}^{1}$. Since

$$
\frac{p \sin g}{\bar{q} \sin f}=\frac{p q}{\sin f \sin g} / \frac{q \bar{q}}{\sin ^{2} g},
$$


$p \sin g /(\bar{q} \sin f)$ is real and negative on $\mathbb{S}^{1}$ if and only if $p q$ is real on $\mathbb{S}^{1}$ and

$$
\frac{p q}{\sin f \sin g}<0 \quad \text { on } \mathbb{S}^{1}
$$

Hence, $L_{1}$ and $L_{2}$ can be simultaneously unitarized via dressing if and only if the conditions of (3.51) are satisfied.

ii) The proof of this part is similar to that of the above part, and the only difference is that here we use Theorem 3.24 instead of Theorem 3.5.

We remark that the conditions (3.51) and (3.52) appear in different forms in [7] and [1] under the additional assumption that $L_{1} L_{2}$ is also unitarizable.

\section{$\S 4$. Simultaneous unitarization of three or more loop matrices}

In this section, we present conditions for the simultaneous unitarizability of three or more loop matrices and show that under these conditions, there is a unique element in $\Lambda_{r, B}^{+} G_{\mathrm{t}}^{\mathbb{C}}$ unitarizing them, unless the loop matrices all commute. As pointed out in the introduction, this uniqueness result will be used in Section 5 to deal with the closing conditions.

Simultaneous unitarization of three loop matrices

First, we consider the simultaneous unitarization of three loop matrices via dressing or $r$-dressing with $r \in(0,1)$. We will see that this case is crucial for the case of four or more loop matrices.

THEOREM 4.1. Let $L_{1}, L_{2}, L_{3} \in \Lambda \boldsymbol{G}_{\mathrm{t}}^{\mathbb{C}} \backslash\{ \pm I\}$ be holomorphic on a neighborhood of $\mathbb{S}^{1}$. Suppose that neither $L_{1}$ and $L_{2}$ nor $L_{2}$ and $L_{3}$ commute.

i) For $i=1,2$ and 3 , assume that $T_{i} \in \Lambda \boldsymbol{G}_{\mathrm{t}}^{\mathbb{C}}$ conjugates $L_{i}$ into $\Lambda^{\mathrm{d}} \boldsymbol{G}_{\mathrm{t}} \cup$ $\Lambda^{\mathrm{a}} \boldsymbol{G}_{\mathrm{t}}$, and define $\widehat{T}_{i}$ from $T_{i}$ as in Section 3 . Set

$$
\widehat{T}_{1} \widehat{T}_{2}^{-1}=\left(\begin{array}{ll}
a & b \\
c & d
\end{array}\right), \quad \widehat{T}_{2} \widehat{T}_{3}^{-1}=\left(\begin{array}{ll}
e & f \\
g & h
\end{array}\right) .
$$

Then, $L_{1}, L_{2}$ and $L_{3}$ can be simultaneously unitarized via dressing if and only if $L_{i}$ and $L_{i+1}$ can be simultaneously unitarized via dressing for $i=1$ and 2 and

$$
\overline{a c e f}=b d g h \quad \text { on } \mathbb{S}^{1} \text {. }
$$


In this case, there is a unique element of $\Lambda_{B}^{+} G_{\mathrm{t}}^{\mathbb{C}}$ simultaneously unitarizing $L_{1}, L_{2}$ and $L_{3}$.

ii) For $i=1,2$ and 3, assume that the two holomorphic eigenvalues of $L_{i}$ are not always equal on $\mathbb{S}^{1}$, let $T_{i}$ be given by (2.7) or (2.8) or (2.9) or (2.12) or (2.13) for $L_{i}$, and define $\widehat{T}_{i}$ from $T_{i}$ as in Section 3 . Then, $L_{1}, L_{2}$ and $L_{3}$ can be simultaneously unitarized via $r$-dressing for some $r \in(0,1)$ sufficiently close to 1 if and only if $L_{i}$ and $L_{i+1}$ can be simultaneously unitarized via $r$-dressing for $i=1$ and 2 and (4.3) is satisfied by the meromorphic functions $a, b, \ldots, h$ defined by (4.2). In this case, there is a unique element of $\Lambda_{r, B}^{+} G_{\mathrm{t}}^{\mathbb{C}}$ simultaneously unitarizing $L_{1}, L_{2}$ and $L_{3}$, and we can choose $r$ such that it can be replaced by any number in $(r, 1)$.

Proof. i) Assume that $L_{i}$ and $L_{i+1}$ can be simultaneously unitarized via dressing for $i=1$ and 2. Then, by Theorem 3.5 and our assumptions,

(4.4) bdef $\not \equiv 0, \frac{\overline{a c}}{b d}$ and $\frac{g h}{\overline{e f}}$ are always finite, real and negative on $\mathbb{S}^{1}$.

By Remark 3.21, the elements of $\Lambda \boldsymbol{G}_{\mathrm{t}}^{\mathbb{C}}$ simultaneously unitarizing $L_{1}$ and $L_{2}$ have the form

$$
U_{\mathrm{L}} T_{*}^{-1}\left(\begin{array}{cc}
\left(-\frac{\overline{a c}}{b d}\right)^{1 / 4} & 0 \\
0 & \left(-\frac{b d}{\overline{a c}}\right)^{1 / 4}
\end{array}\right) T_{*} T_{2} \quad \text { with } U_{\mathrm{L}} \in \Lambda \boldsymbol{G}_{\mathrm{t}}
$$

and the elements of $\Lambda \boldsymbol{G}_{\mathrm{t}}^{\mathbb{C}}$ simultaneously unitarizing $L_{2}$ and $L_{3}$ have the form

$$
U_{\mathrm{R}} T_{*}^{-1}\left(\begin{array}{cc}
\left(-\frac{g h}{\overline{e f}}\right)^{1 / 4} & 0 \\
0 & \left(-\frac{\overline{e f}}{g h}\right)^{1 / 4}
\end{array}\right) T_{*} T_{2} \quad \text { with } U_{\mathrm{R}} \in \Lambda \boldsymbol{G}_{\mathrm{t}}
$$

where in both cases $T_{*}$ is determined by $L_{2}$. Since the matrices given by (4.5) and (4.6) both unitarize $L_{2}$ and the two diagonal matrices in them have positive diagonal entries, the uniqueness of such splitting implies that $L_{1}, L_{2}$ and $L_{3}$ can be simultaneously unitarized via dressing if and only if the two diagonal matrices are equal, i.e., if and only if (4.3) holds.

ii) The proof of this part is similar to that of the above part, but uses Theorem 3.24 and Remark 3.39 in place of Theorem 3.5 and Remark 3.21. 
We would like to point out that the case where $L_{1}$ and $L_{3}$ commute and $T_{3}=T_{1}$ is a special case of the above theorem. In this case, (4.3) follows directly from (3.7) or (3.26).

The following example shows that in general, the simultaneous unitarizability via dressing of every pair of at least three loop matrices does not imply the simultaneous unitarizability via dressing of all the loop matrices.

Example 4.7. Let $r \in(0,1], k>1, l \in(1 / k, 1) \cup(1, k)$ and $t \in(0, \pi)$. Define $T_{2}=I$,

$$
T_{1}(\lambda)=\frac{1}{\sqrt{1+k^{2}}}\left(\begin{array}{cc}
k & \mathrm{i} \lambda \\
\mathrm{i} / \lambda & k
\end{array}\right), T_{3}(\lambda)=\frac{1}{\sqrt{1+k^{2}}}\left(\begin{array}{cc}
k / l & -\mathrm{i} l \lambda \\
-\mathrm{i} / l / \lambda & k l
\end{array}\right)
$$

Consider

$$
L_{1}=T_{1}^{-1} L_{2} T_{1}, \quad L_{2}=\left(\begin{array}{cc}
\mathrm{e}^{\mathrm{i} t} & 0 \\
0 & \mathrm{e}^{-\mathrm{i} t}
\end{array}\right), \quad L_{3}=T_{3}^{-1} L_{2} T_{3} .
$$

Then, for each $i \in\{1,2,3\}, T_{i}$ and $L_{i}$ are in $\Lambda_{r} \boldsymbol{G}_{\mathrm{t}}^{\mathbb{C}}, T_{i}$ diagonalizes $L_{i}$ into $\Lambda_{r} \boldsymbol{G}_{\mathrm{t}}$ (hence $L_{i}$ is in the even case), and the difference of the holomorphic eigenvalues of $L_{i}$ does not have any zero on $\mathbb{S}^{1}$. By Theorems 3.5 and 3.24, from

$$
\begin{aligned}
& T_{1} T_{2}^{-1}=T_{1}, \\
& T_{2} T_{3}^{-1}=\frac{1}{\sqrt{1+k^{2}}}\left(\begin{array}{cc}
k l & \mathrm{i} l \lambda \\
\mathrm{i} / l / \lambda & k / l
\end{array}\right), \\
& T_{1} T_{3}^{-1}=\frac{1}{1+k^{2}}\left(\begin{array}{cc}
k^{2} l-1 / l & \mathrm{i} k(l+1 / l) \lambda \\
\mathrm{i} k(l+1 / l) / \lambda & k^{2} / l-l
\end{array}\right)
\end{aligned}
$$

and our assumptions we see that any two of $L_{1}, L_{2}$ and $L_{3}$ can be simultaneously unitarized via $r$-dressing. However, since

$$
\frac{\overline{k \cdot \mathrm{i} / \lambda}}{\mathrm{i} \lambda \cdot k}=-1, \quad \frac{\mathrm{i} / l / \lambda \cdot k / l}{\overline{k l \cdot \mathrm{i} l \lambda}}=-\frac{1}{l^{4}} \neq-1 \quad \text { on } \mathbb{S}^{1},
$$

$L_{1}, L_{2}$ and $L_{3}$ can not be simultaneously unitarized via $r$-dressing by Theorem 4.1.

Simultaneous unitarization of four or more loop matrices

Finally, we consider the simultaneous unitarization of four or more loop matrices via dressing or $r$-dressing with $r \in(0,1)$. 
TheOrem 4.14. Let $m \geq 4$ be an integer. Assume that $L_{1}, L_{2}, \ldots, L_{m}$ $\in \Lambda \boldsymbol{G}_{\mathrm{t}}^{\mathbb{C}} \backslash\{ \pm I\}$ are holomorphic on a neighborhood of $\mathbb{S}^{1}$.

i) If $L_{1}, L_{2}, \ldots$ and $L_{m}$ can be individually conjugated into $\Lambda^{\mathrm{d}} \boldsymbol{G}_{\mathrm{t}} \cup$ $\Lambda^{\mathrm{a}} \boldsymbol{G}_{\mathrm{t}}$ by elements of $\Lambda \boldsymbol{G}_{\mathrm{t}}^{\mathbb{C}}$, then they can be simultaneously unitarized via dressing if and only if each consecutive triple $L_{i}, L_{i+1}$ and $L_{i+2}$ can be simultaneously unitarized via dressing. In this case, if not all of $L_{1}, L_{2}, \ldots$ and $L_{m}$ commute, then there is a unique element of $\Lambda_{B}^{+} G_{t}^{\mathbb{C}}$ simultaneously unitarizing $L_{1}, L_{2}, \ldots$ and $L_{m}$.

ii) Suppose that for each $i \in\{1,2, \ldots, m\}$, the two holomorphic eigenvalues of $L_{i}$ are not always equal on $\mathbb{S}^{1}$. Then, $L_{1}, L_{2}, \ldots$ and $L_{m}$ can be simultaneously unitarized via $r$-dressing for some $r \in(0,1)$ sufficiently close to 1 if and only if each consecutive triple $L_{i}, L_{i+1}$ and $L_{i+2}$ can be simultaneously unitarized via $r$-dressing. In this case, if not all of $L_{1}, L_{2}, \ldots$ and $L_{m}$ commute, then there is a unique element of $\Lambda_{r, \boldsymbol{B}}^{+} \boldsymbol{G}_{\mathrm{t}}^{\mathbb{C}}$ simultaneously unitarizing $L_{1}, L_{2}, \ldots$ and $L_{m}$, and we can choose $r$ such that it can be replaced by any number in $(r, 1)$.

Proof. i) The necessity is trivial. So, we only need to prove the sufficiency and the uniqueness statement.

We say that two loop matrices $L_{i}$ and $L_{j}$ are equivalent if they commute. Then, this defines an equivalence relation among $L_{1}, L_{2}, \ldots$ and $L_{m}$. By Proposition 3.1, it suffices to consider only one representative from each equivalence class. Thus, we can assume further that every pair of the given loop matrices do not commute. Now assume that $S \in \Lambda_{B}^{+} G_{\mathrm{t}}^{\mathbb{C}}$ unitarizes $L_{1}, L_{2}$ and $L_{3}$, while $R \in \Lambda_{B}^{+} G_{\mathrm{t}}^{\mathbb{C}}$ unitarizes $L_{2}, L_{3}$ and $L_{4}$. Then, the uniqueness statement of Theorem 3.5 yields that $R=S$, since both $R$ and $S$ unitarize $L_{2}$ and $L_{3}$. Hence, $L_{1}, L_{2}, L_{3}$ and $L_{4}$ can be simultaneously unitarized via $R=S$. An iteration of this argument proves the sufficiency and the uniqueness statement of the theorem.

ii) The proof of this part is similar to that of the above part, and the only difference is that here we use Theorem 3.24 instead of Theorem 3.5.

\section{$\S 5$. Pointwise simultaneous unitarization, and closing conditions}

In this section, summarizing our results in Sections 3 and 4, we first state a relation between the simultaneous unitarization of loop matrices via $r$-dressing and the pointwise simultaneous unitarization of the matrices in $\mathrm{SL}(2, \mathbb{C})$. Then, we prove a result concerning the closing conditions to be defined below. 
In Theorems 3.24, 4.1 and 4.14, the conditions for the simultaneous unitarization of two or more loop matrices via $r$-dressing with $r<1$ sufficiently close to 1 are the same as the conditions for the pointwise simultaneous unitarization on $\mathbb{S}^{1}$ of the corresponding matrices in $\operatorname{SL}(2, \mathbb{C})$. Thus, we have the following result.

TheOREM 5.1. Let $m \geq 2$ be an integer. Assume that for each $i \in$ $\{1,2, \ldots, m\}, L_{i} \in \Lambda \boldsymbol{G}_{\mathrm{t}}^{\mathbb{C}} \backslash\{ \pm I\}$ is holomorphic on a neighborhood of $\mathbb{S}^{1}$, and the two holomorphic eigenvalues of $L_{i}$ are not always equal on $\mathbb{S}^{1}$. Then, $L_{1}, L_{2}, \ldots$ and $L_{m}$ can be simultaneously unitarized by $r$-dressing for some $r \in(0,1)$ sufficiently close to 1 if and only if for almost every $\lambda \in \mathbb{S}^{1}$, the matrices $L_{1}(\lambda), L_{2}(\lambda), \ldots$ and $L_{m}(\lambda)$ can be simultaneously unitarized in $\mathrm{SL}(2, \mathbb{C})$. In this case, we can choose $r$ such that it can be replaced by any number in $(r, 1)$.

We note that in [9], a result very similar to Theorem 5.1 is proved by Nick Schmitt using a quite different method.

Let $0<r \leq 1$. Motivated by the construction of CMC surfaces from holomorphic potentials, an $r$-unitary loop matrix $M$ is said to satisfy the closing conditions if

$$
M(\lambda=1)= \pm I, \quad M^{\prime}(\lambda=1)=0 .
$$

We have the following theorem concerning these conditions.

TheOrem 5.3. Let $m \geq 2$ be an integer. Assume that $L_{1}, L_{2}, \ldots, L_{m} \in$ $\Lambda \boldsymbol{G}_{\mathrm{t}}^{\mathbb{C}} \backslash\{ \pm I\}$ are holomorphic on a neighborhood of $\mathbb{S}^{1}$, not all of $L_{1}, L_{2}, \ldots$ and $L_{m}$ commute, there is an $r \in(0,1)$ sufficiently close to 1 and $a T_{+} \in$ $\Lambda_{r}^{+} \boldsymbol{G}_{\mathrm{t}}^{\mathbb{C}}$ simultaneously unitarizing $L_{1}, L_{2}, \ldots$ and $L_{m}$, and the two holomorphic eigenvalues $v_{i}$ and $1 / v_{i}$ of each $L_{i}$ are not always equal on $\mathbb{S}^{1}$. Then, for any $i \in\{1,2, \ldots, m\}$,

i) $T_{+} L_{i} T_{+}^{-1}$ satisfies the closing conditions if and only if

$$
v_{i}(1)= \pm 1, \quad v_{i}^{\prime}(1)=0
$$

ii) when $v_{i}$ can be written as $\mathrm{e}^{\mathrm{i} f_{i}}$ with $f_{i} \in \mathcal{W}$ being real-valued, $T_{+} L_{i} T_{+}^{-1}$ satisfies the closing conditions if and only if

$$
f_{i}(1) \in \mathbb{Z} \pi, \quad f_{i}^{\prime}(1)=0 .
$$


Proof. i) From the proofs and the uniqueness parts of our simultaneous unitarization results in Theorems 3.24, 4.1 and 4.14,

$$
T_{+} L_{i} T_{+}^{-1}=U_{i} T_{*, i}^{-1}\left(\begin{array}{cc}
v_{i} & 0 \\
0 & 1 / v_{i}
\end{array}\right) T_{*, i} U_{i}^{-1}
$$

for some $U_{i} \in \Lambda_{r} \boldsymbol{G}_{\mathrm{t}}$, where $T_{*, i}$ is determined by $L_{i}$ as usual. This implies our claim by the definition (5.2) of the closing conditions.

ii) This part directly follows from Part i).

\section{REFERENCES}

[1] I. Biswas, On the existence of unitary flat connections over the punctured sphere with given local monodromy around the punctures, Asian J. Math., 3 (1999), 333-344.

[2] J. Dorfmeister and G. Haak, On constant mean curvature surfaces with periodic metric, Pacific J. Math., 182 (1998), 229-287.

[3] J. Dorfmeister and G. Haak, Construction of non-simply connected CMC surfaces via dressing, J. Math. Soc. Japan, 55 (2003), 335-364.

[4] J. Dorfmeister, F. Pedit and $\mathrm{H}$. Wu, Weierstrass type representation of harmonic maps into symmetric spaces, Comm. Anal. Geom., 6 (1998), 633-668.

[5] J. Dorfmeister and H. Wu, Construction of constant mean curvature n-noids from holomorphic potentials, Math. Z., to appear.

[6] I. C. Gohberg, A factorization problem in normed rings, functions of isometric and symmetric operators and singular integral equations, Russian Math. Surveys, 19 (1964), 63-114.

[7] W. Goldman, Topological components of spaces of representations, Invent. Math., 93 (1988), 557-607.

[8] Y. Katznelson, An Introduction to Harmonic Analysis, Dover, 1976.

[9] N. Schmitt, Constant mean curvature trinoids, preprint (2001).

Josef Dorfmeister

Department of Mathematics

Tech University of Munich

Garching D-85747

Germany

Hongyou $\mathrm{Wu}$

Department of Mathematics

Northern Illinois University

DeKalb, IL 60115

$U S A$ 